\title{
Searches for pair production of charginos and top squarks in final states with two oppositely charged leptons in proton-proton collisions at $\sqrt{\mathrm{s}}=13 \mathrm{TeV}$
}

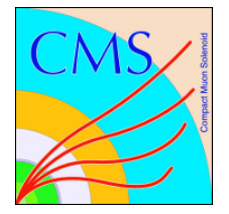

\section{The CMS collaboration}

E-mail: cms-publication-committee-chair@cern.ch

ABSTRACT: A search for pair production of supersymmetric particles in events with two oppositely charged leptons (electrons or muons) and missing transverse momentum is reported. The data sample corresponds to an integrated luminosity of $35.9 \mathrm{fb}^{-1}$ of protonproton collisions at $\sqrt{s}=13 \mathrm{TeV}$ collected with the CMS detector during the 2016 data taking period at the LHC. No significant deviation is observed from the predicted standard model background. The results are interpreted in terms of several simplified models for chargino and top squark pair production, assuming $R$-parity conservation and with the neutralino as the lightest supersymmetric particle. When the chargino is assumed to undergo a cascade decay through sleptons, with a slepton mass equal to the average of the chargino and neutralino masses, exclusion limits at $95 \%$ confidence level are set on the masses of the chargino and neutralino up to 800 and $320 \mathrm{GeV}$, respectively. For top squark pair production, the search focuses on models with a small mass difference between the top squark and the lightest neutralino. When the top squark decays into an off-shell top quark and a neutralino, the limits extend up to 420 and $360 \mathrm{GeV}$ for the top squark and neutralino masses, respectively.

KEYwords: Hadron-Hadron scattering (experiments), Supersymmetry

ArXiv EPrint: 1807.07799 


\section{Contents}

1 Introduction 1

2 The CMS detector $\quad 3$

3 Data and simulated samples 3

4 Event reconstruction 5

$\begin{array}{lll}5 & \text { Search strategy } & 7\end{array}$

6 Background estimation $\quad 10$

6.1 Modeling of $m_{\mathrm{T} 2}(\ell \ell)$ in $\mathrm{t} \overline{\mathrm{t}}, \mathrm{tW}$, and WW events 11

6.2 Normalization of tEZ, WZ, ZZ, and Drell-Yan backgrounds 11

$\begin{array}{lll}7 & \text { Systematic uncertainties } & 13\end{array}$

8 Results and interpretation $\quad 15$

9 Summary 22

$\begin{array}{ll}\text { The CMS collaboration } & 31\end{array}$

\section{Introduction}

The standard model (SM) of particle physics has so far been able to describe a wide variety of phenomena with outstanding precision. However, the SM does not address the hierarchy problem between the Higgs boson mass and the Planck scale [1, 2], and does not contain a dark matter candidate to explain cosmological observations [3-5]. Supersymmetry [614] is an extension of the SM that assigns a fermion (boson) superpartner to every SM boson (fermion). This theory can solve the hierarchy problem since the large quantum loop corrections to the Higgs boson mass, due mainly to the top quark, can be largely canceled by the analogous corrections from the top quark superpartner [15-17]. Moreover, if $R$-parity [18] is conserved, the lightest supersymmetric particle (LSP) is stable and, if massive, provides a good candidate for dark matter.

This paper presents a search for supersymmetric particle production in final states with two oppositely charged (OC) leptons $(\ell)$ and missing transverse momentum stemming from the two LSPs. Only electrons (e) and muons $(\mu)$ are considered. The search targets two specific signal scenarios with chargino $\left(\widetilde{\chi}_{1}^{ \pm}\right)$and top squark $\left(\widetilde{\mathrm{t}}_{1}\right)$ pair production, using data from proton-proton (pp) collisions at $\sqrt{s}=13 \mathrm{TeV}$ collected by the CMS experiment [19] at the CERN LHC in 2016, and corresponding to an integrated luminosity of $35.9 \mathrm{fb}^{-1}$.

The results are interpreted in terms of simplified supersymmetric model spectra (SMS) [20-22] scenarios. The search for chargino pair production considers, as a reference, a model (figure 1, left) where the charginos decay into a lepton, a neutrino $(\nu)$, and 

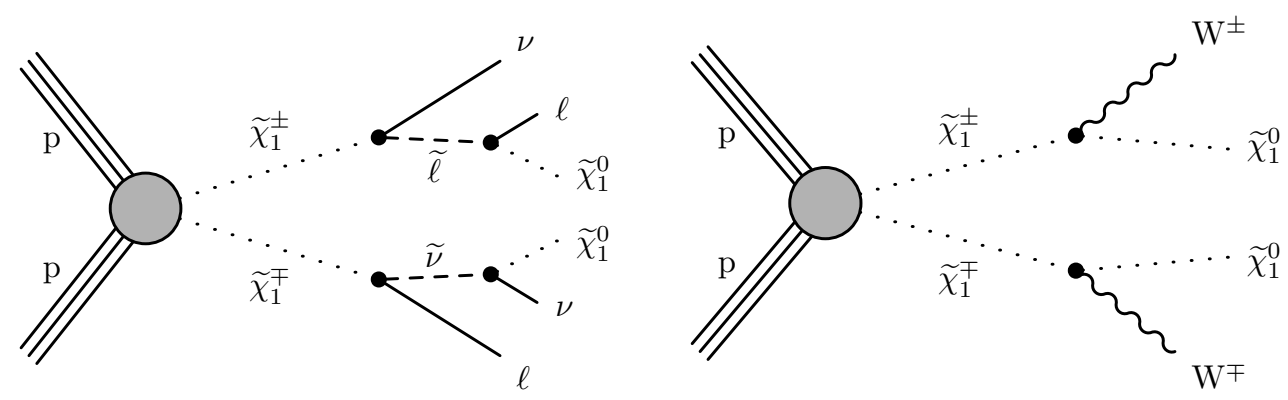

Figure 1. Simplified-model diagrams of chargino pair production with two benchmark decay modes: the left plot shows decays through intermediate sleptons or sneutrinos, while the right one displays prompt decays into a $\mathrm{W}$ boson and the lightest neutralino.

the lightest neutralino $\left(\widetilde{\chi}_{1}^{0}\right)$ via an intermediate charged slepton $\left(\widetilde{\chi}_{1}^{ \pm} \rightarrow \nu \widetilde{\ell} \rightarrow \nu \ell \widetilde{\chi}_{1}^{0}\right)$ or sneutrino $\left(\widetilde{\chi}_{1}^{ \pm} \rightarrow \ell \widetilde{\nu} \rightarrow \ell \nu \widetilde{\chi}_{1}^{0}\right)$. The three generations of sleptons are assumed to be degenerate, with a mass equal to the average of the chargino and neutralino masses. The branching fractions ( $\mathcal{B}$ 's) of the chargino decays into charged sleptons or sneutrinos are assumed to be equal. Results are also interpreted in terms of a second model (figure 1, right), where each chargino decays into the lightest neutralino and a $\mathrm{W}$ boson. Searches for chargino pair production have been previously published by the CMS Collaboration in the context of the former scenario using $8 \mathrm{TeV}$ collision data [23] and by the ATLAS Collaboration in the context of both scenarios using $8 \mathrm{TeV}$ [24-26] and $13 \mathrm{TeV}$ [27-29] collision data.

The search for top squark pair production focuses on an SMS in which the top squark decays into a top quark and the lightest neutralino as shown in figure 2 (left). The analysis strategy is optimized for a compressed spectrum scenario where the mass difference $(\Delta m)$ between the top squark and the lightest neutralino lies between the top quark and $\mathrm{W}$ boson masses $m_{\mathrm{W}}<\Delta m \lesssim m_{\mathrm{t}}$. In this regime, the top quarks are produced off-shell, giving rise to final states with low-momentum bottom quarks which often fail to be identified. Further interpretations of the results are given in terms of an additional model, where each of the pair-produced top squarks decays into a bottom quark and a chargino, which in turn decays into a $\mathrm{W}$ boson and the lightest neutralino, as shown in figure 2 (right). In this model, the mass of the chargino is assumed to be equal to the average of the top squark and neutralino masses. This work is complementary to another OC dilepton search published by the CMS Collaboration [30], aimed at testing models where $\Delta m>m_{\mathrm{t}}$, which result in signatures with on-shell top quarks and higher momentum particles. With respect to that analysis, this search gains sensitivity in the compressed mass region by loosening the requirements on the jets from bottom quark hadronization and optimizing the signal event selection for the lower momentum carried by the neutralino LSPs. The CMS Collaboration has also published other searches targeting the same signal models in the final states with exactly one lepton [31] and with no leptons [32], with the latter also covering the four-body-decay of the top squark in the region $\Delta m<80 \mathrm{GeV}$. The ATLAS Collaboration published several searches addressing these signal models using all three final states [33-35].

The paper is organized as follows: section 2 introduces the experimental apparatus; sections 3 and 4 describe the data and simulated event samples used in this search and 

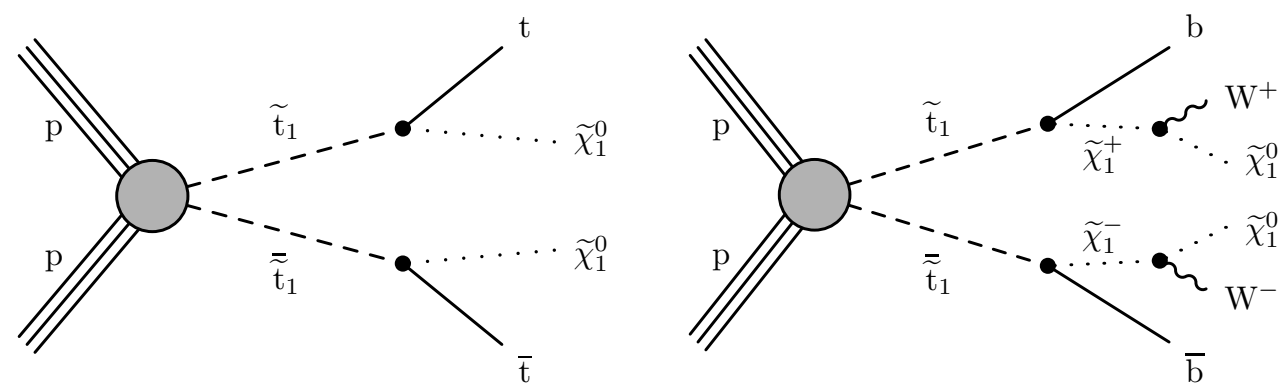

Figure 2. Simplified-model diagrams of top squark pair production with two benchmark decay modes of the top squark: the left plot shows decays into a top quark and the lightest neutralino, while the right one displays prompt decays into a bottom quark and a chargino, further decaying into a neutralino and a $\mathrm{W}$ boson.

the details on the reconstruction of the physics objects, respectively; section 5 presents the general strategy of the analysis; section 6 discusses the estimates of the contributions from SM processes to the selected events; section 7 details the sources of systematic uncertainties for signal and background processes; section 8 reports the results and their interpretation in terms of the considered SMS; and finally section 9 summarizes the results of the search.

\section{The CMS detector}

The central feature of the CMS apparatus is a superconducting solenoid of $6 \mathrm{~m}$ internal diameter, providing a magnetic field of $3.8 \mathrm{~T}$. In the inner part of the solenoid volume is a silicon pixel and strip tracker, which reconstructs the trajectories of the charged particles up to a pseudorapidity $|\eta|<2.5$. Outside the tracker, a lead tungstate crystal electromagnetic calorimeter (ECAL) and a brass and scintillator hadron calorimeter (HCAL), each composed of a barrel and two endcap sections, measure the energy of the particles in the region $|\eta|<3$. Forward calorimeters extend coverage provided by the barrel and endcap detectors up to $|\eta|<5$. The information from the tracker and calorimeter systems is merged to reconstruct electrons and hadronic jets. Muons are detected in gas-ionization chambers embedded in the steel flux-return yoke outside the solenoid, covering the region $|\eta|<2.4$. The detector is nearly hermetic, allowing for momentum balance measurements in the plane transverse to the beam direction. A more detailed description of the CMS detector, together with a definition of the coordinate system used and the relevant kinematic variables, can be found in ref. [19].

\section{Data and simulated samples}

Events of interest are selected using triggers [36] which require the presence of two leptons (ee, $\mu \mu, \mathrm{e} \mu$ ). The threshold on the transverse momentum $\left(p_{\mathrm{T}}\right)$ of the leading lepton is $23 \mathrm{GeV}$ for the ee and e $\mu$ triggers, and $17 \mathrm{GeV}$ for the $\mu \mu$ triggers. The threshold for the trailing lepton is 8 (12) GeV for muons (electrons). To increase the efficiency of the trigger selection, events are also accepted by triggers requiring at least one electron (muon) with $p_{\mathrm{T}}>25(24) \mathrm{GeV}$, passing tighter identification criteria than the ones applied in the double-lepton triggers. The trigger performances are measured with leptons from $\mathrm{Z} \rightarrow \ell^{+} \ell^{-}$ 
decays. The combined efficiency of the dilepton and single-lepton triggers for signal events is found to range between 90 and $99 \%$, depending on the $p_{\mathrm{T}}$ and $\eta$ of the leptons.

Samples of Monte Carlo (MC) simulated events are used to study the contribution of SM processes to the selected data set and the expected acceptance for the different signal models. Events from top quark-antiquark pair $(\mathrm{t} \overline{\mathrm{t}})$ production are generated with POWHEG v2 [37-39] and normalized to the expected cross section calculated at next-tonext-to-leading order (NNLO) in perturbative quantum chromodynamics (QCD), including resummation of next-to-next-to-leading logarithmic (NNLL) soft gluon terms [40]. Events with a single top quark produced in association with a $\mathrm{W}$ boson (tW) are generated with POWHEG v1 [41] and normalized to an approximate NNLO cross section calculation [42]. Diboson production (WW, WZ, and ZZ) via quark-antiquark annihilation is simulated at next-to-leading order (NLO) using POWHEG v2 [43, 44]. The yields of events from WW production are scaled to the NNLO cross section [45]. Events from $\mathrm{q} \overline{\mathrm{q}} \rightarrow \mathrm{ZZ}$ production are reweighted via NNLO/NLO $K$ factors, as functions of the generated ZZ system mass [46]. Two additional sets of $K$ factors, as functions of the generated ZZ system $p_{\mathrm{T}}$ and of the azimuthal separation $(\Delta \phi)$ between the $\mathrm{Z}$ bosons, are used to evaluate the uncertainty in the kinematic properties of $\mathrm{ZZ}$ production. Diboson production via gluon fusion is simulated using MCFM v7 [47], and LO cross sections obtained from the generator are corrected with the NNLO/LO $K$ factors [46, 48]. Drell-Yan events are generated with MADGRAPH5_aMC@NLO v2.2.2 [49] at LO, and event yields are scaled to the NNLO cross

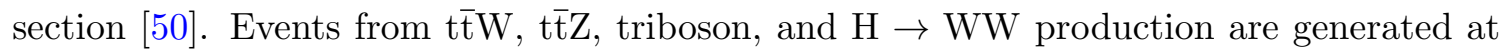
NLO $[51,52]$ with the MADGRAPH5_aMC@NLO generator.

Chargino pair production and top squark pair production events are generated using MADGRAPH5_amC@NLO at LO with up to two extra partons in the matrix element calculations, and are normalized to the respective cross sections computed at NLO plus next-to-leading logarithmic (NLL) precision [53-61], with all the other sparticles assumed to be heavy and decoupled. In the case of chargino pair production, calculations are performed in a limit of mass-degenerate wino $\widetilde{\chi}_{2}^{0}$ and $\widetilde{\chi}_{1}^{ \pm}$, and light bino $\widetilde{\chi}_{1}^{0}$.

All processes are generated using the NNPDF3.0 [62] parton distribution function (PDF) set. The parton showering, hadronization, and the underlying event are modeled using PYTHIA 8.212 [63] with the CUETP8M1 [64] underlying event tune for all the processes, except in the generation of $t \bar{t}$ events, where the first emission is done at the matrix element level with POWHEG v2 and the CUETP8M2T4 [65] tune is used. Weights for the estimation of theoretical systematic uncertainties, including those related to the choice of PDFs, and renormalization and factorization scales, are included in simulated events [66].

The detector response to the generated events is simulated using a realistic model of the CMS detector based on GEANT4 [67] for SM processes, while for signal events a fast simulation (FASTSIM) [68] of the detector based on a parametrization of the average response to particles is used. Simulated events are subsequently reconstructed using the same algorithms as applied to data.

In order to model the effect of multiple interactions per bunch crossing (pileup), simulated events are mixed with minimum-bias events simulated with PYTHIA, and are reweighted in order to match the observed rate of multiple interactions. 
The modeling and normalization of the main background processes are studied in data, as discussed in section 6 . The modeling of $\mathrm{t} \overline{\mathrm{t}}, \mathrm{tW}$, and $\mathrm{WW}$ production is studied in data control regions (CRs), and their normalization is determined via a maximum like-

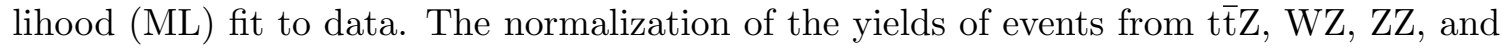
Drell-Yan production is taken from simulation and corrected by the event rates measured in dedicated CRs.

To improve the modeling of jets from initial-state radiation (ISR) in simulated signal events, reweighting factors are applied, which make the distributions of observables for related SM processes in simulation agree with control samples in data. For chargino pair production, mediated by the electroweak interaction, the reweighting procedure is based on studies of $p_{\mathrm{T}}$ balance in inclusive $\mathrm{Z}$ boson production events [69]. Events are then reweighted according to the total transverse momentum $\left(p_{\mathrm{T}}^{\mathrm{ISR}}\right)$ of the system of supersymmetric particles. The reweighting factors range between 1.18 at $p_{\mathrm{T}}^{\mathrm{ISR}} \approx 125 \mathrm{GeV}$ and 0.78 for $p_{\mathrm{T}}^{\mathrm{ISR}}>600 \mathrm{GeV}$. A global reweighting is further applied in order not to alter the signal production cross section. As top squark pair production occurs via strong interactions, a different set of reweighting factors is derived as a function of the multiplicity of ISR jets $\left(N_{\mathrm{jet}}^{\mathrm{ISR}}\right)$ in a sample of $\mathrm{t} \overline{\mathrm{t}}$ events selected by requiring an OC electron-muon pair and two jets identified as coming from bottom quark hadronization. The measured reweighting factors vary between 0.92 and 0.51 for $N_{\text {jet }}^{\text {ISR }}$ between 1 and 6 , with an additional scale factor applied to keep the total event yields invariant.

\section{Event reconstruction}

The particle-flow algorithm [70] aims to reconstruct and identify each individual particle in an event, with an optimized combination of information from the various elements of the CMS detector. The energy of photons is directly obtained from the ECAL measurement, corrected for zero-suppression effects. The energy of electrons is determined from a combination of the electron momentum at the primary interaction vertex as determined by the tracker, the energy of the corresponding ECAL cluster, and the energy sum of all bremsstrahlung photons spatially compatible with originating from the electron track. The momentum of muons is obtained from the curvature of the corresponding track. The energy of charged hadrons is determined from a combination of their momentum measured in the tracker and the matching ECAL and HCAL energy deposits, corrected for zero-suppression effects and for the response function of the calorimeters to hadronic showers. Finally, the energy of neutral hadrons is obtained from the corresponding corrected ECAL and HCAL energy.

The reconstructed vertex with the largest value of summed physics-object $p_{\mathrm{T}}^{2}$ is taken to be the primary pp interaction vertex. The physics objects are the jets, clustered using a jet finding algorithm [71, 72] with the tracks assigned to the vertex as inputs, and the associated momentum imbalance in the transverse plane, taken as the negative vector $p_{\mathrm{T}}$ sum of those jets.

The identification of the muons used in the analysis is based on the number of reconstructed energy deposits in the tracker and in the muon system, and on the fit quality of 
the muon track [73]. Electron identification relies on quality criteria of the electron track, matching between the electron trajectory and the associated cluster in the calorimeter, and shape observables of the electromagnetic shower observed in the ECAL [74]. The efficiency for the reconstruction and selection of the muons (electrons) is found to be 70-95 (30-75)\% depending on their $p_{\mathrm{T}}$ and $\eta$.

The lepton selection is further optimized to select leptons from the decays of $\mathrm{W}$ or $\mathrm{Z}$ bosons. The leptons are required to be isolated by measuring their relative isolation $\left(I_{\text {rel }}\right)$, as the ratio of the scalar $p_{\mathrm{T}}$ sum of the photons and of the neutral and charged hadrons within a cone of radius $R=\sqrt{(\Delta \phi)^{2}+(\Delta \eta)^{2}}=0.3$ around the candidate lepton, and the $p_{\mathrm{T}}$ of the lepton itself. The contribution of particles produced in pileup interactions is reduced by considering only charged hadrons consistent with originating from the primary vertex of the event, and correcting for the expected contribution of neutral hadrons from the pileup [73, 74]. Leptons are considered to be isolated if their relative isolation $I_{\text {rel }}$ is found to be smaller than 0.12. A looser requirement of $I_{\text {rel }}<0.4$ is used to define a veto lepton selection. Candidate lepton trajectories are further required to be compatible with the primary interaction vertex by imposing constraints on their transverse $\left(d_{0}\right)$ and longitudinal $\left(d_{z}\right)$ impact parameters, and on the three-dimensional impact parameter significance $\left(S_{3 \mathrm{D}}^{\mathrm{d}}\right)$, computed as the ratio of the three-dimensional impact parameter and its uncertainty. Both electrons and muons are required to satisfy the conditions $\left|d_{0}\right|<0.05 \mathrm{~cm},\left|d_{z}\right|<$ $0.10 \mathrm{~cm}$, and $S_{3 \mathrm{D}}^{\mathrm{d}}<4$. Finally, electrons originating from photon conversions are rejected by requiring that the electron track not have missing hits in the innermost layers of the tracker, and not form a conversion vertex with any other candidate electron in the event [74].

For each event, hadronic jets are clustered from the PF reconstructed particles using the infrared and collinear-safe anti- $k_{\mathrm{T}}$ algorithm [71, 72] with a distance parameter of 0.4. The jet momentum is determined as the vectorial sum of all particle momenta in the jet, and is found in the simulation to be within 5 to $10 \%$ of its true value over the whole $p_{\mathrm{T}}$ spectrum and detector acceptance. Jet energy corrections are derived from simulation to bring the measured response of jets to that of particle level jets on average. In situ measurements of the momentum balance in the dijet, multijet, photon+jet, and leptonically decaying $\mathrm{Z}+$ jet events are used to account for any residual difference in jet energy scale in data and simulation [75]. Additional quality criteria are applied to reject spurious jets from detector noise. Finally, the jets overlapping with any selected lepton within a cone of radius $R<0.4$ are removed.

Jets originating from the hadronization of bottom quarks ( $b$ jets) are identified by the combined secondary vertex v2 b-tagging algorithm, using the medium operating point [76]. This requirement provides an efficiency for identifying b jets that increases from 50 to $70 \%$ for jets with $p_{\mathrm{T}}$ from 20 to $100 \mathrm{GeV}$. The misidentification rate for jets originating from light quarks and gluons is about $1 \%$ in the same $p_{\mathrm{T}}$ range.

The momentum imbalance of the event in the transverse plane is referred to as missing transverse momentum $\left(\vec{p}_{\mathrm{T}}^{\text {miss }}\right)$ and it is defined as the negative vectorial $p_{\mathrm{T}}$ sum of all PF candidates in the event, taking into account the energy corrections applied to the jets [77]. The magnitude of $\vec{p}_{\mathrm{T}}^{\text {miss }}$ is denoted as $p_{\mathrm{T}}^{\text {miss }}$.

Differences have been observed in the modeling of the $\vec{p}_{\mathrm{T}}^{\text {miss }}$ resolution in events simulated with FASTSim and with the full detector simulation. To account for this effect, the 
acceptance for signal events is computed both using the $\vec{p}_{\mathrm{T}}^{\text {miss }}$ at the generator level and after the event reconstruction. The average value of the two acceptances in each analysis bin is taken as the central value for the acceptance.

Simulated events are reweighted to account for differences with respect to data in the efficiencies of the lepton reconstruction, identification, and isolation requirements, and in the performance of b-jet identification. The values of the data-to-simulation scale factors differ from unity by less than $10 \%$ with typical efficiency corrections of $2-3$ (5)\% for the identification of leptons (b jets) with $p_{\mathrm{T}}>20 \mathrm{GeV}$ and $|\eta|<2.4$.

\section{Search strategy}

The search strategy is developed for two signal hypotheses: the chargino pair and top squark pair productions. The first signal hypothesis is studied along the whole $\left(m_{\widetilde{\chi}_{1}^{ \pm}}, m_{\widetilde{\chi}_{1}^{0}}\right)$ mass plane, while for the second one the analysis is optimized on the compressed scenario, where the mass difference of the top squark and the lightest neutralino is in between the top quark and $\mathrm{W}$ boson masses. The searches involve the same techniques for the background estimation and the signal extraction, while they differ slightly in the signal region (SR) selection in order to improve their respective sensitivity.

The signal models are characterized by a common final state with two OC leptons and two lightest neutralinos contributing to large $p_{\mathrm{T}}^{\text {miss }}$. Based on this, a general highacceptance baseline selection is defined, requiring two OC isolated leptons with $|\eta|<2.4$ and $p_{\mathrm{T}} \geq 25(20) \mathrm{GeV}$ for the leading (trailing) lepton. Events with $\tau$ leptons decaying into electrons or muons that satisfy the selection requirements are taken into account. To reduce the contributions from low-mass resonances, $\mathrm{Z} \rightarrow \tau \tau$ production, and nonprompt leptons from hadronic jets, the invariant mass $m_{\ell \ell}$ of the lepton pair is required to be greater than $20 \mathrm{GeV}$, and if both leptons have the same flavor (SF), $m_{\ell \ell}$ is further required to satisfy $\left|m_{\ell \ell}-m_{\mathrm{Z}}\right|>15 \mathrm{GeV}$, where $m_{\mathrm{Z}}$ is the $\mathrm{Z}$ boson mass. High $p_{\mathrm{T}}^{\text {miss }}(\geq 140 \mathrm{GeV})$ is required. Events are further rejected if they contain a third lepton with $p_{\mathrm{T}}>15 \mathrm{GeV}$, $|\eta|<2.4$, and satisfying the veto lepton selection (as detailed in section 4). A summary of the baseline selection is found in table 1 .

The SM processes that contribute most after the baseline selection are $t \overline{\mathrm{t}}$, $\mathrm{tW}$, and $\mathrm{WW}$ production. For all these backgrounds, the lepton pair and $\vec{p}_{\mathrm{T}}^{\text {miss }}$ come from a W boson pair. Consequently, the variable $m_{\mathrm{T} 2}$ [78] is constructed to generalize the transverse mass $\left(m_{\mathrm{T}}\right)$ for a system with two invisible particles, by using the two leptons as the two visible systems,

$$
m_{\mathrm{T} 2}(\ell \ell)=\min _{\vec{p}_{\mathrm{T}}^{\text {miss1 }}+\vec{p}_{\mathrm{T}}^{\text {miss2 }}=\vec{p}_{\mathrm{T}}^{\text {miss }}}\left(\max \left[m_{\mathrm{T}}\left(\vec{p}_{\mathrm{T}}^{\text {lep1 }}, \vec{p}_{\mathrm{T}}^{\text {miss1 } 1}\right), m_{\mathrm{T}}\left(\vec{p}_{\mathrm{T}}^{\text {lep2 }}, \vec{p}_{\mathrm{T}}^{\text {miss2 }}\right)\right]\right) .
$$

This observable reaches a kinematic endpoint at the $m_{\mathrm{W}}$ for the considered backgrounds. Signal events, instead, present $m_{\mathrm{T} 2}(\ell \ell)$ spectra without such an endpoint because of the additional contribution to the $\vec{p}_{\mathrm{T}}^{\text {miss }}$ given by the neutralinos. The sensitivity of the analysis is further enhanced by dividing the SR in bins of $p_{\mathrm{T}}^{\text {miss }}$. This allows the analysis not only to exploit the larger tails in the $p_{\mathrm{T}}^{\text {miss }}$ distribution of the signal events, but also to optimize the 


\begin{tabular}{|lc|}
\hline Variable & Selection \\
\hline Lepton flavor & $\mathrm{e}^{+} \mathrm{e}^{-}, \mu^{+} \mu^{-}, \mathrm{e}^{ \pm} \mu^{\mp}$ \\
Leading lepton & $p_{\mathrm{T}} \geq 25 \mathrm{GeV},|\eta|<2.4$ \\
Trailing lepton & $p_{\mathrm{T}} \geq 20 \mathrm{GeV},|\eta|<2.4$ \\
Third lepton veto & $p_{\mathrm{T}} \geq 15 \mathrm{GeV},|\eta|<2.4$ \\
$m_{\ell \ell}$ & $\geq 20 \mathrm{GeV}$ \\
$\left|m_{\ell \ell}-m_{\mathrm{Z}}\right|$ & $>15 \mathrm{GeV}$ only for ee and $\mu \mu$ events \\
$p_{\mathrm{T}}^{\text {miss }}$ & $\geq 140 \mathrm{GeV}$ \\
\hline
\end{tabular}

Table 1. Definition of the baseline selection used in the searches for chargino and top squark pair production.

sensitivity to signals with different mass separation between the produced supersymmetric particle and the LSP. Each $p_{\mathrm{T}}^{\text {miss }}$ bin is in turn divided into events with SF and different flavor (DF) leptons to exploit the smaller contamination from WZ, ZZ, and Drell-Yan production of the latter.

The SRs are further subdivided based on the specific characteristics of each signal model. A veto on b-tagged jets is applied to reject $t \bar{t}, t W$, and $t \bar{t} Z$ events in the chargino search. Selected events in the $p_{\mathrm{T}}^{\text {miss }}$ bins below $300 \mathrm{GeV}$ are then split into two different subregions, depending on the presence of a jet with $p_{\mathrm{T}}>20 \mathrm{GeV}$ and $|\eta|<2.4$. This allows for a better discrimination between signal events and top quark background, which still contaminates the SRs after applying the b-tagged jet veto. Events with b-tagged jets are kept as a $\mathrm{CR}$ for the normalization of the background from t $\overline{\mathrm{t}}$ and $\mathrm{tW}$ production (discussed in section 6).

The final states produced in the top squark decays are characterized by the presence of two bottom quarks. When the difference in the mass of the top squark and the neutralino is close to the edge of the compressed region, $\Delta m \gtrsim m_{\mathrm{W}}$, the bottom quarks are soft and give rise to jets with relatively low momentum that have a lower probability to be tagged. In this case, the top squark final states are similar to those from chargino pair production, and requiring a veto on b-tagged jets is again an effective strategy to define SRs with reduced contamination from $t \bar{t}, t W$, and $t \bar{t} Z$ backgrounds. For signal scenarios with larger $\Delta m$, instead, the b jets have higher momentum and the final states are more $\mathrm{t} \overline{\mathrm{t}}$-like. Consequently, sensitivity to top squark production is enhanced by requiring a b-tagged jet to reduce the background from diboson and Drell-Yan events.

Another useful means to discriminate top squark production from SM processes is given by the presence of high- $p_{\mathrm{T}}$ jets from ISR in the events. The invisible particles (neutrinos and neutralinos) produced in the decay chain of the top squark in the compressed scenario are expected to be soft; events with harder neutralinos, however, can arise when the top squark pair system recoils against a high- $p_{\mathrm{T}}$ ISR jet. In this hard ISR regime, background is still constrained by the kinematic $m_{\mathrm{W}}$ endpoint in $m_{\mathrm{T} 2}(\ell \ell)$, and can be effectively separated from the signal. Hard ISR events are selected by requiring that the leading jet satisfies $p_{\mathrm{T}}>150 \mathrm{GeV}$ and is not $\mathrm{b}$ tagged. In order to favor the topology in which the jet recoils 


\begin{tabular}{|lcccccccc|}
\hline & $\mathrm{SR} 1_{\text {Otag }}^{\text {jet }}$ & $\mathrm{SR} 1_{\text {0tag }}^{\text {jets }}$ & $\mathrm{CR} 1_{\text {tags }}$ & $\mathrm{SR} 2_{\text {0tag }}^{\text {jet }}$ & $\mathrm{SR} 2_{\text {0ta }}^{\text {jets }}$ & $\mathrm{CR} 2_{\text {tags }}$ & $\mathrm{SR} 3_{0 \text { tag }}$ & $\mathrm{CR} 3_{\text {tags }}$ \\
\hline$p_{\mathrm{T}}^{\text {miss }}[\mathrm{GeV}]$ & $140-200$ & $140-200$ & $140-200$ & $200-300$ & $200-300$ & $200-300$ & $\geq 300$ & $\geq 300$ \\
$N_{\mathrm{b} \text { jets }}$ & 0 & 0 & $\geq 1$ & 0 & 0 & $\geq 1$ & 0 & $\geq 1$ \\
$N_{\text {jets }}$ & 0 & $\geq 1$ & $\geq 1$ & 0 & $\geq 1$ & $\geq 1$ & $\geq 0$ & $\geq 1$ \\
Channels & $\mathrm{SF}, \mathrm{DF}$ & $\mathrm{SF}, \mathrm{DF}$ & $\mathrm{SF}, \mathrm{DF}$ & $\mathrm{SF}, \mathrm{DF}$ & $\mathrm{SF}, \mathrm{DF}$ & $\mathrm{SF}, \mathrm{DF}$ & $\mathrm{SF}, \mathrm{DF}$ & $\mathrm{SF}, \mathrm{DF}$ \\
$m_{\mathrm{T} 2}(\ell \ell)$ & $0-20,20-40,40-60,60-80,80-100,100-120, \geq 120 \mathrm{GeV}$ & \\
\hline
\end{tabular}

Table 2. Definition of the SRs for the chargino search as a function of the $p_{\mathrm{T}}^{\text {miss }}$ value, the bjet multiplicity and jet multiplicity. Also shown are the CRs with b-tagged jets used for the normalization of the t $\bar{t}$ and tW backgrounds. Each of the regions is further divided in seven $m_{\mathrm{T} 2}(\ell \ell)$ bins as described in the last row.

\begin{tabular}{|lcccccc|}
\hline & $\mathrm{SR}_{0 \text { tag }}$ & $\mathrm{SR}_{\text {tags }}$ & $\mathrm{SR}_{0 \text { tag }}$ & $\mathrm{SR} 2_{\text {tags }}$ & $\mathrm{SR} 3_{\text {0tag }}^{\mathrm{ISR}}$ & $\mathrm{SR} 3_{\text {tag }}^{\text {ISR }}$ \\
\hline$p_{\mathrm{T}}^{\text {miss }}[\mathrm{GeV}]$ & $140-200$ & $140-200$ & $200-300$ & $200-300$ & $\geq 300$ & $\geq 300$ \\
$N_{\mathrm{b} \text { jets }}$ & 0 & $\geq 1$ & 0 & $\geq 1$ & 0 & $\geq 1$ \\
$N_{\text {jets }}$ & $\geq 0$ & $\geq 1$ & $\geq 0$ & $\geq 1$ & $\geq 1$ & $\geq 2$ \\
ISR jets & $\geq 0$ & $\geq 0$ & $\geq 0$ & $\geq 0$ & $\geq 1$ & $\geq 1$ \\
Channels & $\mathrm{SF}, \mathrm{DF}$ & $\mathrm{SF}, \mathrm{DF}$ & $\mathrm{SF}, \mathrm{DF}$ & $\mathrm{SF}, \mathrm{DF}$ & $\mathrm{SF}, \mathrm{DF}$ & $\mathrm{SF}, \mathrm{DF}$ \\
$m_{\mathrm{T} 2}(\ell \ell)$ & $0-20,20-40,40-60,60-80,80-100,100-120, \geq 120 \mathrm{GeV}$ \\
\hline
\end{tabular}

Table 3. Definition of the $\mathrm{SRs}$ for top squark production search as a function of the $p_{\mathrm{T}}^{\text {miss }}$ value, the b-jet multiplicity and the ISR jet requirement. Each of the regions is further divided in seven $m_{\mathrm{T} 2}(\ell \ell)$ bins as described in the last row.

against the rest of the system, the $\Delta \phi$ between the jet and the $\vec{p}_{\mathrm{T}}^{\text {miss }}$ is required to be larger than 2.5 rad. This requirement is found to be effective in discriminating top squark production from background events at high $p_{\mathrm{T}}^{\text {miss }}$, and is therefore applied only for events with $p_{\mathrm{T}}^{\text {miss }}>300 \mathrm{GeV}$.

A summary of the SRs for the chargino and top squark searches is given in tables 2 and 3 , respectively, indicating the $p_{\mathrm{T}}^{\text {miss }}$ range, the selection on the multiplicity of jets $\left(N_{\text {jets }}\right)$ and b jets $\left(N_{\mathrm{b}}\right.$ jets $)$ in the event, and the ISR jet requirement. The observed distributions of some observables used to define the SRs are compared to SM expectations in figure 3.

Each of the SRs defined in tables 2 and 3 is further divided into seven $m_{\mathrm{T} 2}(\ell \ell)$ bins of $20 \mathrm{GeV}$ width, starting from $0 \mathrm{GeV}$ and with the last bin collecting all events with $m_{\mathrm{T} 2}(\ell \ell)>120 \mathrm{GeV}$. A simultaneous ML fit to the $m_{\mathrm{T} 2}(\ell \ell)$ distribution in all the SRs is then performed to extract the signal (as described in section 8). Since the first $m_{\mathrm{T} 2}(\ell \ell$ ) bins have a low signal contribution, we exploit them to constrain the contributions of the dominant backgrounds in the SRs with one b-tagged jet (dominated by t $\overline{\mathrm{t}}$ and $\mathrm{tW}$ production) and without b-tagged jets (where WW production becomes relevant) through the fit. 

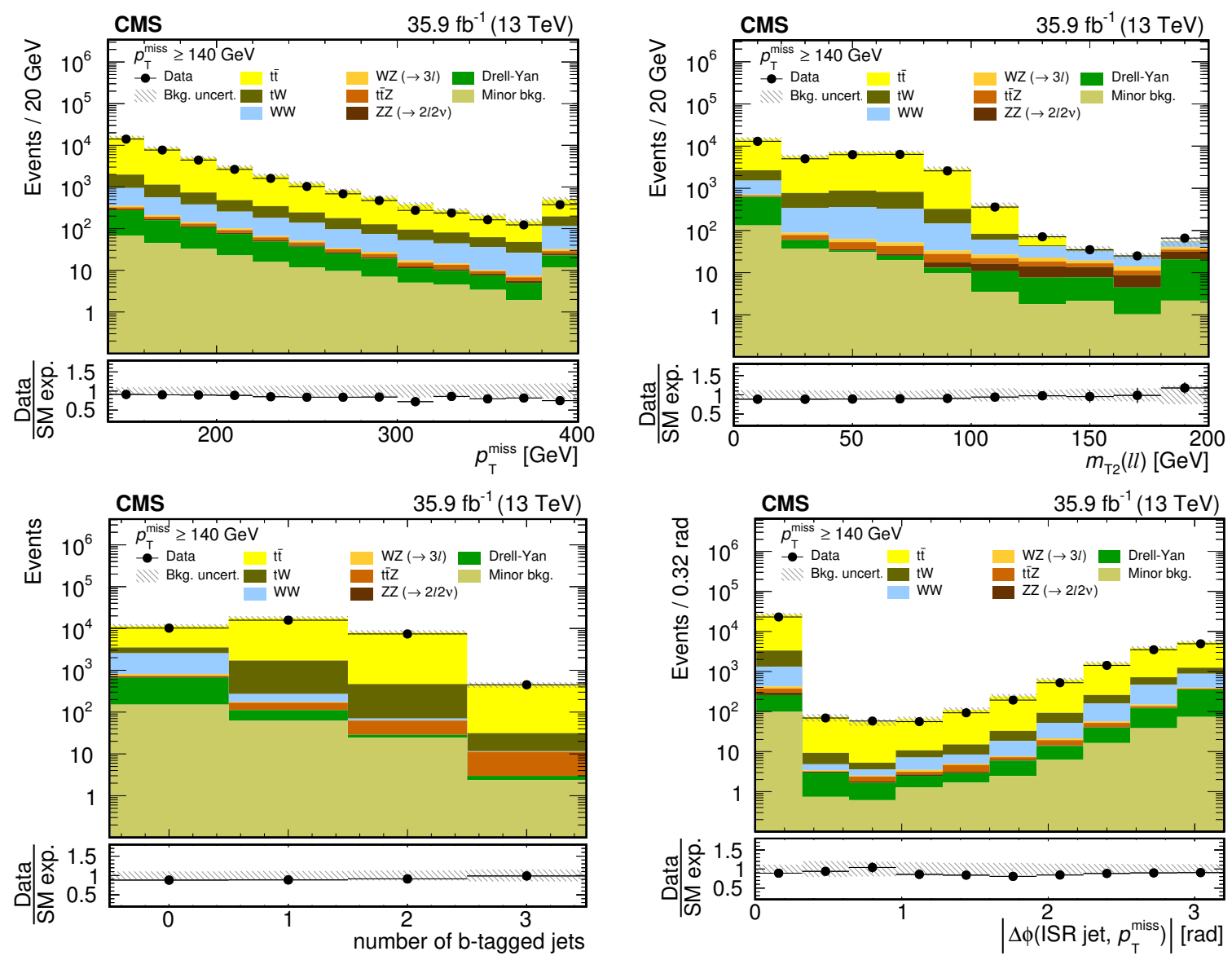

Figure 3. Observed and SM expected distributions of some observables used to define the SRs for events with two OC isolated leptons and $p_{\mathrm{T}}^{\text {miss }} \geq 140 \mathrm{GeV}$. Clockwise from top left: $p_{\mathrm{T}}^{\text {miss }}, m_{\mathrm{T} 2}(\ell \ell)$, $\Delta \phi$ between the $\vec{p}_{\mathrm{T}}^{\text {miss }}$ and the leading jet (required not to be b-tagged and with $p_{\mathrm{T}}>150 \mathrm{GeV}$, events missing this requirements are shown in the first bin), and multiplicity of b-tagged jets in the event. The last bin includes the overflow entries. The contributions of minor backgrounds such as $\mathrm{t} \overline{\mathrm{t}} \mathrm{W}, \mathrm{H} \rightarrow \mathrm{WW}$, and triboson production are grouped together. In the bottom panel, the ratio of observed and expected yields is shown. The hatched band represents the total uncertainty in the background expectation, as described in section 7 .

\section{Background estimation}

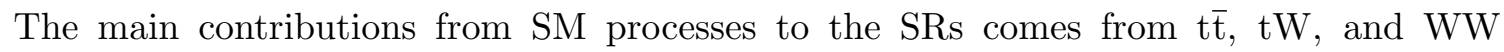
production. The normalization of these backgrounds is determined by the ML fit, as mentioned in section 5 . Their $m_{\mathrm{T} 2}(\ell \ell)$ shape has a natural endpoint at the $m_{\mathrm{W}}$, and events enter into the relevant region for signal extraction $\left(m_{\mathrm{T} 2}(\ell \ell)>80 \mathrm{GeV}\right)$ mainly due to detector resolution effects, whose contributions are not easy to model. For this reason, we study the modeling of the $m_{\mathrm{T} 2}(\ell \ell)$ distribution for these processes in dedicated CRs in data described in section 6.1. The contributions of the subleading t€Z, WZ, ZZ, and Drell-Yan backgrounds are also tested in CRs, where correction factors for their normalizations are extracted, as discussed in section 6.2. Remaining minor backgrounds from $\mathrm{t} \overline{\mathrm{t}} \mathrm{W}, \mathrm{H} \rightarrow \mathrm{WW}$, 
and triboson production give small contributions in the SRs, and the estimates for these processes are taken directly from simulation. Background contributions from rest of the SM processes are found to be negligible. The contribution of signal to any of the CRs used is found to be negligible compared to SM processes.

\subsection{Modeling of $m_{\mathrm{T} 2}(\ell \ell)$ in $\mathrm{t} \overline{\mathrm{t}}, \mathrm{tW}$, and $\mathrm{WW}$ events}

The simulated $m_{\mathrm{T} 2}(\ell \ell)$ distributions for $\mathrm{t} \overline{\mathrm{t}}$, $\mathrm{tW}$, and $\mathrm{WW}$ backgrounds are validated in two CRs. To construct the first one, the baseline selection is modified by requiring $100<$

$p_{\mathrm{T}}^{\text {miss }}<140 \mathrm{GeV}$. The events in this $\mathrm{CR}$ are further separated according to their b-jet multiplicity to define two sub-regions with different content in top quark ( $t \bar{t}$ and $t W$ ) and WW backgrounds. In order to reject events from Drell-Yan production, only DF events are considered. The second $\mathrm{CR}$ aims at validating the modeling of the $m_{\mathrm{T} 2}(\ell \ell)$ distributions in events with $p_{\mathrm{T}}^{\text {miss }}>140 \mathrm{GeV}$. For this purpose, we select events from $\mathrm{WZ} \rightarrow 3 \ell 1 \nu$ production and emulate the $m_{\mathrm{T} 2}(\ell \ell)$ shape of $\mathrm{WW}$ and top quark events. We take the lepton from the $\mathrm{Z}$ boson with the same charge as the lepton from the $\mathrm{W}$ boson, and we add its $p_{\mathrm{T}}$ vectorially to $\vec{p}_{\mathrm{T}}^{\text {miss }}$, effectively treating it like a neutrino. These events are selected by requiring three leptons and vetoing the presence of a fourth lepton passing the veto lepton requirements. A veto is applied to events with b-tagged jets to remove residual t $\bar{t}$ events. Among the three leptons, a pair of OC SF leptons with an invariant mass within $10 \mathrm{GeV}$ of the $\mathrm{Z}$ boson mass is required to identify the $\mathrm{Z}$ boson. The simulation is found to describe the data well in the CRs. Based on the statistical precision of these CRs, a conservative uncertainty of $5,10,20$, and $30 \%$ is taken for the bins $60 \leq m_{\mathrm{T} 2}(\ell \ell)<80 \mathrm{GeV}, 80 \leq m_{\mathrm{T} 2}(\ell \ell)<100 \mathrm{GeV}, 100 \leq m_{\mathrm{T} 2}(\ell \ell)<120 \mathrm{GeV}$, and $m_{\mathrm{T} 2}(\ell \ell) \geq 120 \mathrm{GeV}$, respectively. These uncertainties are applied to top quark and WW production, and treated as uncorrelated between the two types of backgrounds.

Another potential source of mismodeling in the tails of the $m_{\mathrm{T} 2}(\ell \ell)$ distributions arises from nonprompt leptons originating, for instance, from semileptonic decays of B hadrons in $\mathrm{b}$ jets or from hadronic jets accidentally passing the lepton selection. The value of $m_{\mathrm{T} 2}(\ell \ell)$ in $\mathrm{t} \overline{\mathrm{t}}, \mathrm{tW}$, and $\mathrm{WW}$ events with one nonprompt lepton replacing a prompt one failing the selection requirements will not be bound by the $m_{\mathrm{W}}$ endpoint. The contribution of these events is found to be less than $1 \%$ of the expected background across the different SRs. It becomes more relevant only at large values of $m_{\mathrm{T} 2}(\ell \ell)$ and $p_{\mathrm{T}}^{\text {miss }}$, where it constitutes up to $20 \%$ of the têt background. We study the modeling of the rate of nonprompt leptons in simulation by selecting events with two leptons with the same charge and at least one b-tagged jet. The dominant contribution to this sample comes from $t \bar{t}$ events with a nonprompt lepton. Based on the observed agreement with data, a correction factor of $1.08 \pm 0.21$ is derived for the nonprompt lepton rate in simulation.

\subsection{Normalization of $\mathrm{t} \overline{\mathrm{tZ}}, \mathrm{WZ}, \mathrm{ZZ}$, and Drell-Yan backgrounds}

The production of $t \bar{t} Z$ events where the two $\mathrm{W}$ bosons decay leptonically and the $\mathrm{Z}$ boson decays into neutrinos leads to final states with the same experimental signature as the signal events and with no natural endpoint for the reconstructed $m_{\mathrm{T} 2}(\ell \ell)$ distribution, due to the additional contribution of the neutrinos from the $\mathrm{Z}$ boson decay to the $\vec{p}_{\mathrm{T}}^{\text {miss }}$. The 


\begin{tabular}{|lccc|}
\hline \multirow{3}{*}{ Process } & \multicolumn{3}{c|}{ Scale factors } \\
& $N_{\text {jets }}=0(\mathrm{a})$ & $N_{\text {jets }}>0(\mathrm{a})$ & $N_{\text {jets }} \geq 0(\mathrm{~b})$ \\
\hline $\mathrm{t} \overline{\mathrm{tZ}}$ & $1.44 \pm 0.36$ & $1.44 \pm 0.36$ & $1.44 \pm 0.36$ \\
$\mathrm{WZ}$ & $0.97 \pm 0.09$ & $0.97 \pm 0.09$ & $0.97 \pm 0.09$ \\
$\mathrm{ZZ}$ & $0.74 \pm 0.19$ & $1.21 \pm 0.17$ & $1.05 \pm 0.12$ \\
\hline
\end{tabular}

Table 4. Summary of the normalization scale factors for $t \bar{t} Z$, WZ, and ZZ backgrounds in the SRs used for the chargino (a) and top squark (b) searches. Uncertainties include the statistical uncertainties of data and simulated event samples, and the systematic uncertainties on the number of expected events from the residual processes in the CRs.

normalization of this background is validated in events with three leptons, $p_{\mathrm{T}}^{\text {miss }}>140 \mathrm{GeV}$, and at least two jets with $p_{\mathrm{T}}>20 \mathrm{GeV}$, of which at least one is tagged as b jet. At least one pair of OC SF leptons with an invariant mass not further than $10 \mathrm{GeV}$ from the $\mathrm{Z}$ boson mass is also required. A normalization scale factor of $1.44 \pm 0.36$ for $\mathrm{t} \overline{\mathrm{t} Z}$ production is measured comparing the observed and predicted numbers of events.

Events from WZ production enter the signal event selection when both bosons decay leptonically and one of the three decay leptons fails the veto lepton requirements. We test the modeling of this source of background in a CR with three leptons, $p_{\mathrm{T}}^{\text {miss }}>140 \mathrm{GeV}$, and no b-tagged jets, and derive a normalization scale factor of $0.97 \pm 0.09$ for the simulated WZ background.

The ZZ background is dominated by events with one boson decaying into charged leptons and the other one decaying into neutrinos. This contribution is studied by mimicking the $\mathrm{ZZ} \rightarrow 2 \ell 2 \nu$ production via $\mathrm{ZZ} \rightarrow 4 \ell$ events, where the $p_{\mathrm{T}}$ of one of the reconstructed $\mathrm{Z}$ bosons (randomly chosen between the ones satisfying the $\left|m_{\ell \ell}-m_{Z}\right|<15 \mathrm{GeV}$ condition) is added to the $\vec{p}_{\mathrm{T}}^{\text {miss }}$. Events are selected by requiring four leptons, with one lepton allowed to pass the looser veto lepton requirement in order to increase the acceptance for ZZ production. The events are retained if the four leptons can be arranged into two pairs of OC SF leptons, both with an invariant mass within $30 \mathrm{GeV}$ of the $\mathrm{Z}$ boson mass, and at least one within $15 \mathrm{GeV}$. A scale factor for the $\mathrm{ZZ}$ background normalization is derived in events with $p_{\mathrm{T}}^{\text {miss }}>140 \mathrm{GeV}$ and with no b-tagged jets. Since the chargino search uses separate SRs for events with or without jets, two corresponding scale factors are also measured, which suggest a higher jet multiplicity in data than in ZZ simulated events.

A summary of the scale factors derived in this section is given in table 4. For all the quoted scale factors, uncertainties include the statistical uncertainties on data and simulated events, and the systematic uncertainties on the number of expected events from the residual processes in the CRs.

Drell-Yan events can pass the baseline selection because of mismeasurements in $p_{\mathrm{T}}^{\text {miss }}$. We study the modeling of this background in events with two OC SF leptons with $\mid m_{\ell \ell}-$ $m_{\mathrm{Z}} \mid<15 \mathrm{GeV}$, no additional leptons, and no b-tagged jets ( $\mathrm{Z}$ boson events). The events with $100<p_{\mathrm{T}}^{\text {miss }}<140 \mathrm{GeV}$ are dominated by Drell-Yan production, and are used to derive a $m_{\mathrm{T} 2}(\ell \ell)$ shape correction, which is subsequently tested in $\mathrm{Z}$ boson events with 
$p_{\mathrm{T}}^{\text {miss }}>140 \mathrm{GeV}$. The correction ranges from a few percent at low $m_{\mathrm{T} 2}(\ell \ell)$ to about $50 \%$ for $m_{\mathrm{T} 2}(\ell \ell)>100 \mathrm{GeV}$. An overall normalization uncertainty of $32 \%$ is also established by the observed disagreement between data and simulated events with $100<p_{\mathrm{T}}^{\text {miss }}<140 \mathrm{GeV}$. Finally, the predictions for Drell-Yan events with no jets are tested in $\mathrm{Z}$ boson events with no jets and $p_{\mathrm{T}}^{\text {miss }}>140 \mathrm{GeV}$ : a conservative uncertainty of $100 \%$ in this contribution is applied. The Drell-Yan production is a subdominant background in the SRs with no jets and this uncertainty has a negligible impact on the expected sensitivity for signal production.

\section{Systematic uncertainties}

Several sources of systematic uncertainty that affect both the normalizations and the $m_{\mathrm{T} 2}(\ell \ell)$ shapes of the background and signal events are considered in the analysis.

- The overall uncertainty in the integrated luminosity is estimated to be $2.5 \%$ [79].

- The uncertainty on the measured trigger efficiency is $2 \%$.

- Lepton identification and isolation efficiencies are corrected by data-to-simulation scale factors measured in $\mathrm{Z} \rightarrow \ell \ell$ events. The corresponding uncertainties are typically smaller than $3 \%$ per lepton.

- The jet energy scale is varied by its uncertainty [75], and the changes are propagated to all the related observables in the event.

- The energy scale of the low- $p_{\mathrm{T}}$ particles that are not clustered in jets is varied by its uncertainty, and the changes are propagated to the $\vec{p}_{\mathrm{T}}^{\text {miss }}$.

- The efficiencies and misidentification rates of the b-jet identification algorithms are also corrected by data-to-simulation scale factors measured in inclusive jet and t $\overline{\mathrm{t}}$ events [76]. The respective uncertainties range between 1 and $6 \%$, depending on the $p_{\mathrm{T}}$ and $\eta$ of the jets.

- The effect of the simulated data sample sizes on the modeling of the $m_{\mathrm{T} 2}(\ell \ell)$ distributions is taken into account by treating the statistical uncertainty in each bin for each process as an additional uncorrelated uncertainty.

- Uncertainties in the renormalization and factorization scales, and PDFs are propagated by taking the largest changes in the acceptance when independently doubling and halving the renormalization and factorization scales, and when varying the choice of PDFs between the NNPDF3.0 replicas. The PDF uncertainties are not considered for signal models as they are found to be redundant, once the uncertainty in the ISR modeling is included.

The estimates of the SM backgrounds are also affected by specific uncertainties in the modeling of the different processes. 
- A background normalization uncertainty is applied for each background separately. The normalizations of the $\mathrm{t} \overline{\mathrm{t}}, \mathrm{tW}$, and WW processes are determined by the ML fit, as described in section 8 . We assign a common normalization parameter for $t \bar{t}$ and tW events and another for WW production. No explicit normalization uncertainty is defined for $\mathrm{t} \overline{\mathrm{t}}$ and WW events, while a $10 \%$ uncertainty is set for the tW process to

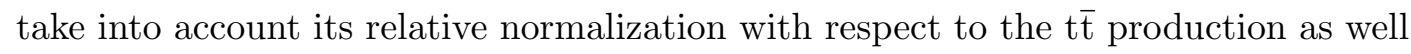

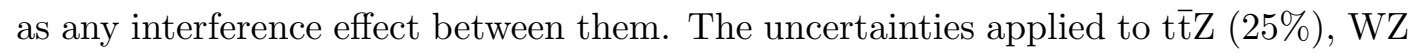
(9\%), and ZZ (26\% in the SRs with 0 jets, $14 \%$ in the SRs with at least 1 jet, and $11 \%$ in the rest of the SRs) correspond to the scale factor uncertainties obtained in section 6.2. Minor backgrounds ( $\mathrm{t} \overline{\mathrm{t}} \mathrm{W}, \mathrm{H} \rightarrow \mathrm{WW}$, triboson production) are assigned a conservative uncertainty of 50\%. Finally, Drell-Yan events have a 100\% normalization uncertainty in the SR with no jets and $32 \%$ in all other SRs.

- The modeling of the yields of events with no jets has been explicitly studied in section 6.2 for ZZ and Drell-Yan production. For the other SM processes, we introduce a related uncertainty by adding two free parameters in the ML fit, scaling respectively the rate of events with no jets for diboson and b-enriched $(\mathrm{t} \overline{\mathrm{t}}, \mathrm{tW}$, $\mathrm{t} \overline{\mathrm{t} Z}$, and $\mathrm{t} \overline{\mathrm{t}} \mathrm{W})$ backgrounds. The total number of expected events without b-tagged jets is constrained to remain invariant, so that only a migration of events between the SRs with and without jets is allowed.

- The modeling of the $m_{\mathrm{T} 2}(\ell \ell)$ shapes in events with an endpoint at the $m_{\mathrm{W}}(\mathrm{t} \overline{\mathrm{t}}, \mathrm{tW}$, and WW) has been studied in section 6.1: an uncertainty of 5, 10, 20, and $30 \%$ is assigned for the last four $m_{\mathrm{T} 2}(\ell)$ bins.

- The choice of the set of NNLO/NLO $K$ factors applied to the $\mathrm{q} \overline{\mathrm{q}} \rightarrow \mathrm{ZZ}$ events affects the modeling of the $m_{\mathrm{T} 2}(\ell \ell)$ shape for the $\mathrm{ZZ}$ background (as described in section 3). Relative variations range from $16 \%$ for $m_{\mathrm{T} 2}(\ell \ell)<20 \mathrm{GeV}$ to about $2 \%$ for $m_{\mathrm{T} 2}(\ell \ell)>120 \mathrm{GeV}$ and are taken as the uncertainties.

- The $m_{\mathrm{T} 2}(\ell \ell)$ distribution in Drell-Yan events has been corrected by scale factors derived in bins of $m_{\mathrm{T} 2}(\ell \ell)$ in the validation region $100<p_{\mathrm{T}}^{\text {miss }}<140 \mathrm{GeV}$, as discussed in section 6.2. The full size of the correction in each bin is taken as an uncertainty.

- The weight of events with nonprompt leptons in simulated samples is varied by the $\pm 19 \%$ uncertainty in the correction factor derived in events with two same-charge leptons, as described in section 6.1.

- The spectrum of top quark $p_{\mathrm{T}}$ in t $\overline{\mathrm{t}}$ events has been observed to be softer in data than in simulated events [80-82]. An uncertainty is derived from the observed variations when reweighting the $\mathrm{t} \overline{\mathrm{t}}$ events to the $p_{\mathrm{T}}$ distribution observed in data.

Finally, additional uncertainties in the modeling of signal events are taken into account, mostly related to the performance of the event reconstruction in FASTSIM.

- The uncertainty in the lepton identification efficiency in events simulated with FASTSim, relative to the full detector simulation, is estimated to be $2 \%$. 
- The analogous uncertainty in the b-tagging efficiency in FASTSim samples ranges between $0.2-0.5 \%$.

- The $\vec{p}_{\mathrm{T}}^{\text {miss }}$ modeling in FASTSim events is studied by comparing the acceptances computed using the $\vec{p}_{\mathrm{T}}^{\text {miss }}$ at the generator level and after the event reconstruction. Since the average of the two is taken as central value for the acceptance, half of their difference is taken as an uncertainty, fully correlated among bins.

- An uncertainty in the modeling of pileup events in FASTSim signal samples is derived by studying the dependence of the acceptance on the multiplicity of primary vertices reconstructed in the event. This uncertainty varies from 0 to $10 \%$ across the SRs and $m_{\mathrm{T} 2}(\ell \ell)$ bins.

- Simulated signal events are reweighted to improve the modeling of the ISR, as described in section 3. Uncertainties on the reweighting procedure are derived from closure tests. For chargino models, the deviation from unity is taken as the systematic uncertainty in the $p_{\mathrm{T}}^{\mathrm{ISR}}$ reweighting factors. For top squark models, half of the deviation from unity in the $N_{\text {jet }}^{\text {ISR }}$ factors is taken.

Tables 5 and 6 summarize the systematic uncertainties in the predicted yields for SM processes and for two reference signals, respectively.

\section{Results and interpretation}

A simultaneous binned ML fit to the $m_{\mathrm{T} 2}(\ell \ell)$ distribution in all the SRs is performed. Uncertainties due to signal and background normalizations are included through nuisance parameters with log-normal prior distributions, while uncertainties in the shape of the $m_{\mathrm{T} 2}(\ell \ell)$ distributions are included with Gaussian prior distributions. As explained in section 6 , the normalizations of the main backgrounds from top quark and WW production are left to be determined in the fit via the constraint provided by the low $m_{\mathrm{T} 2}(\ell \ell)$ region with and without b-tagged jets. The results of the fit in the SRs for the chargino search are shown in figures 4 and 5 for DF and SF events, respectively. The results for the top squark search are shown in figures 6 and 7. Each figure compares the number of observed events in the SRs with the expected yields from SM processes after a background-only fit. As a comparison, the expected yields for a representative signal point are given. The total expected SM contributions before the fit and after a background+signal fit are also shown. Detailed information on the observed and expected yields after the background-only fit are given in tables 7-8 for all dilepton final states and all SRs. No excess over SM prediction is observed in data. The asymptotic approximation of the $\mathrm{CL}_{\mathrm{s}}$ criterion [83-85] is used to set upper limits at $95 \%$ confidence level (CL) on the production cross sections for the different signal models considered.

The 95\% CL upper limits on chargino pair production cross sections with the chargino decaying into sleptons are shown in figure 8 (left). The $\widetilde{\chi}_{1}^{ \pm} \rightarrow \nu \widetilde{\ell} \rightarrow \nu \ell \widetilde{\chi}_{1}^{0}$ and $\widetilde{\chi}_{1}^{ \pm} \rightarrow \ell \widetilde{\nu} \rightarrow$ $\ell \nu \widetilde{\chi}_{1}^{0}$ decay chains are given a $\mathcal{B}$ of $50 \%$ each, and the sleptons are assumed to be degenerate, with a mass equal to the average of the chargino and neutralino masses. By comparing the 

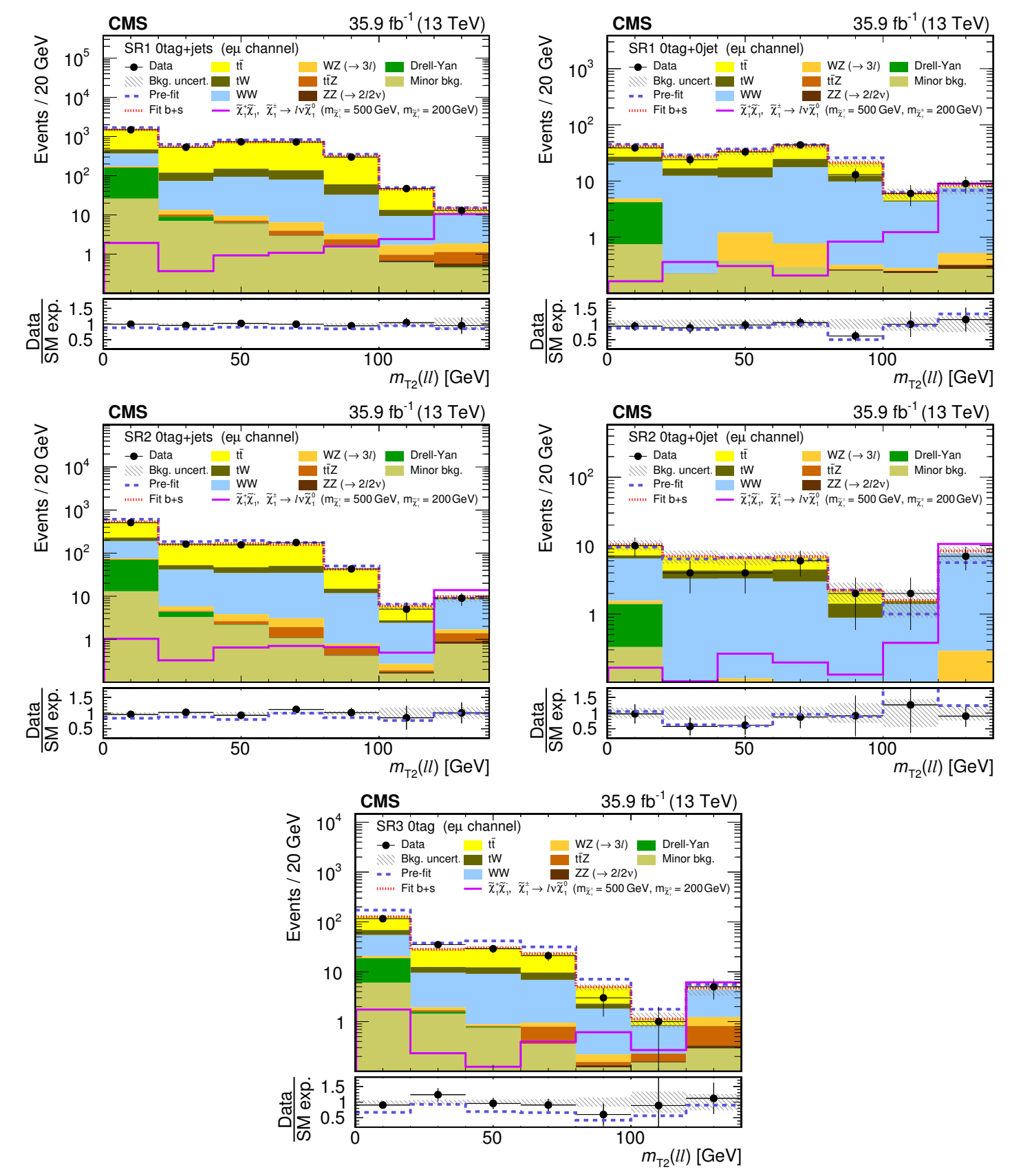

Figure 4. Distributions of $m_{\mathrm{T} 2}(\ell \ell)$ after the fit to data in the chargino SRs with $140<p_{\mathrm{T}}^{\text {miss }}<$ $200 \mathrm{GeV}$ (upper plots), $200<p_{\mathrm{T}}^{\text {miss }}<300 \mathrm{GeV}$ (middle), and $p_{\mathrm{T}}^{\text {miss }}>300 \mathrm{GeV}$ (lower), for $\mathrm{DF}$ events without b-tagged jets and at least one jet (left plots) and no jets (right plots). The lower plot for the SR with $p_{\mathrm{T}}^{\text {miss }}>300 \mathrm{GeV}$ shows all the events without b-tagged jets regardless of their jet multiplicity. The solid magenta histogram shows the expected $m_{\mathrm{T} 2}(\ell \ell)$ distribution for chargino pair production with $m_{\tilde{\chi}_{1}^{ \pm}}=500 \mathrm{GeV}$ and $m_{\widetilde{\chi}_{1}^{0}}=200 \mathrm{GeV}$. Expected total SM contributions before the fit (dark blue dashed line) and after a background+signal fit (dark red dotted line) are also shown. The last bin includes the overflow entries. In the bottom panel, the ratio of data and SM expectations is shown for the expected total SM contribution after the fit using the background-only hypothesis (black dots) and before any fit (dark blue dashed line). The hatched band represents the total uncertainty after the fit. 

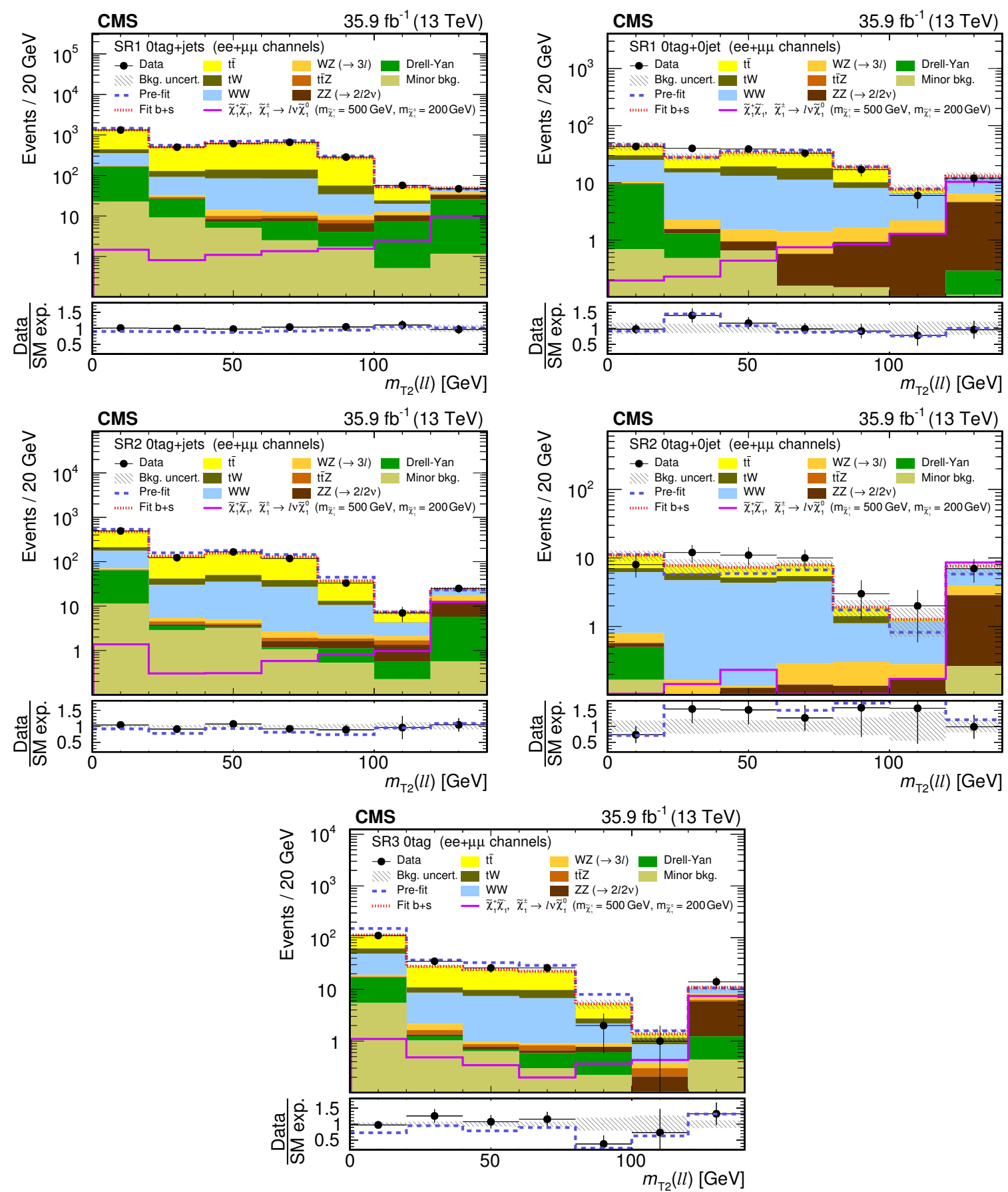

Figure 5. The same distributions of $m_{\mathrm{T} 2}(\ell \ell)$ as figure 4 , but for SF events. 

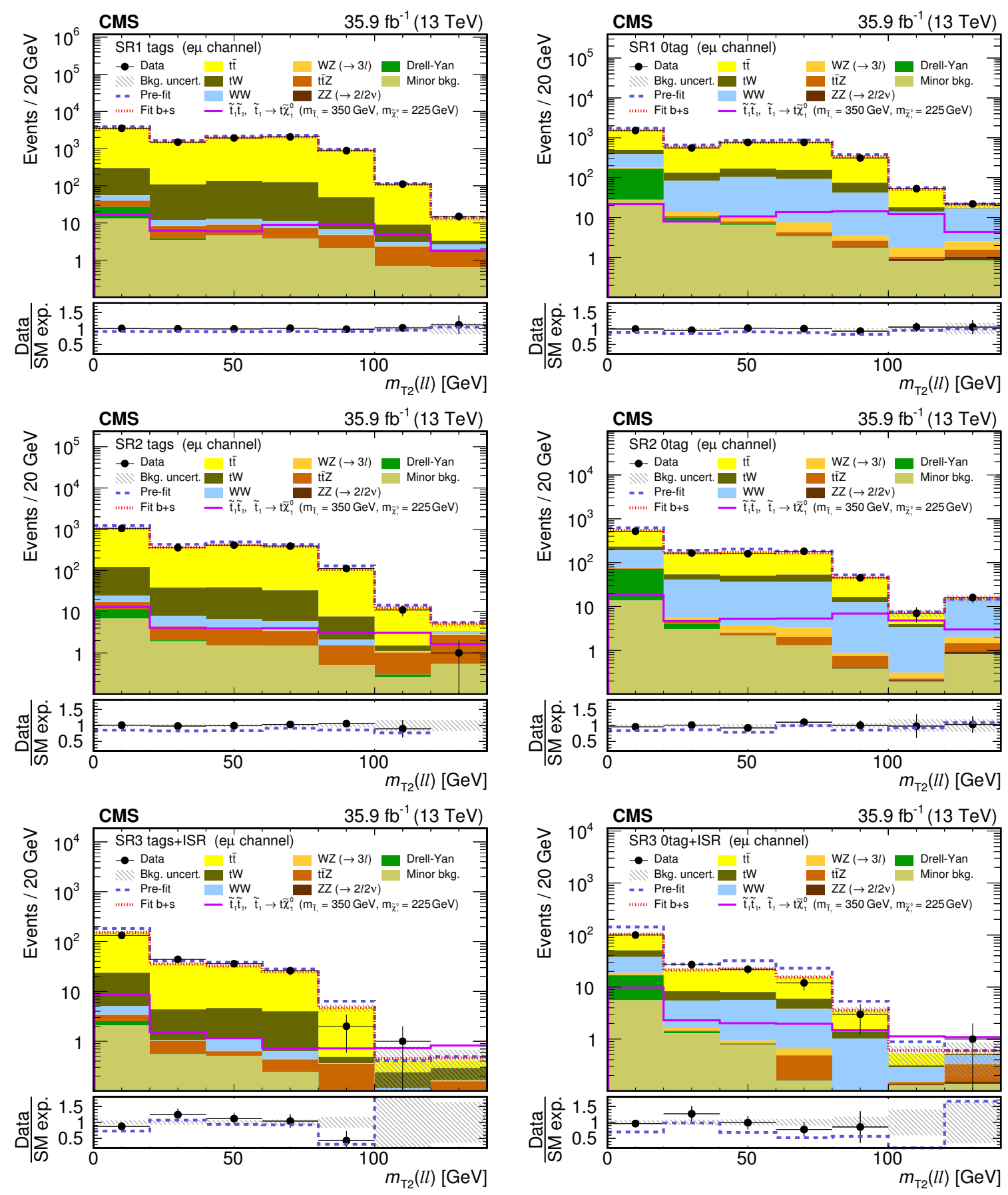

Figure 6. Distributions of $m_{\mathrm{T} 2}(\ell \ell)$ after the fit to data in the top squark SRs with $140<p_{\mathrm{T}}^{\text {miss }}<$ $200 \mathrm{GeV}$ (upper plots), $200<p_{\mathrm{T}}^{\text {miss }}<300 \mathrm{GeV}$ (middle), or $p_{\mathrm{T}}^{\text {miss }}>300 \mathrm{GeV}$ (lower), for DF events with b-tagged jets (left plots) and without b-tagged jets (right plots). The solid magenta histogram shows the expected $m_{\mathrm{T} 2}(\ell \ell)$ distribution for top squark pair production with $m_{\widetilde{\mathrm{t}}_{1}}=350 \mathrm{GeV}$ and $m_{\widetilde{\chi}_{1}^{0}}=225 \mathrm{GeV}$. Expected total SM contributions before the fit (dark blue dashed line) and after a background+signal fit (dark red dotted line) are also shown. The last bin includes the overflow entries. In the bottom panel, the ratio of data and SM expectations is shown for the expected total SM contribution after the fit using the background-only hypothesis (black dots) and before any fit (dark blue dashed line). The hatched band represents the total uncertainty after the fit. 

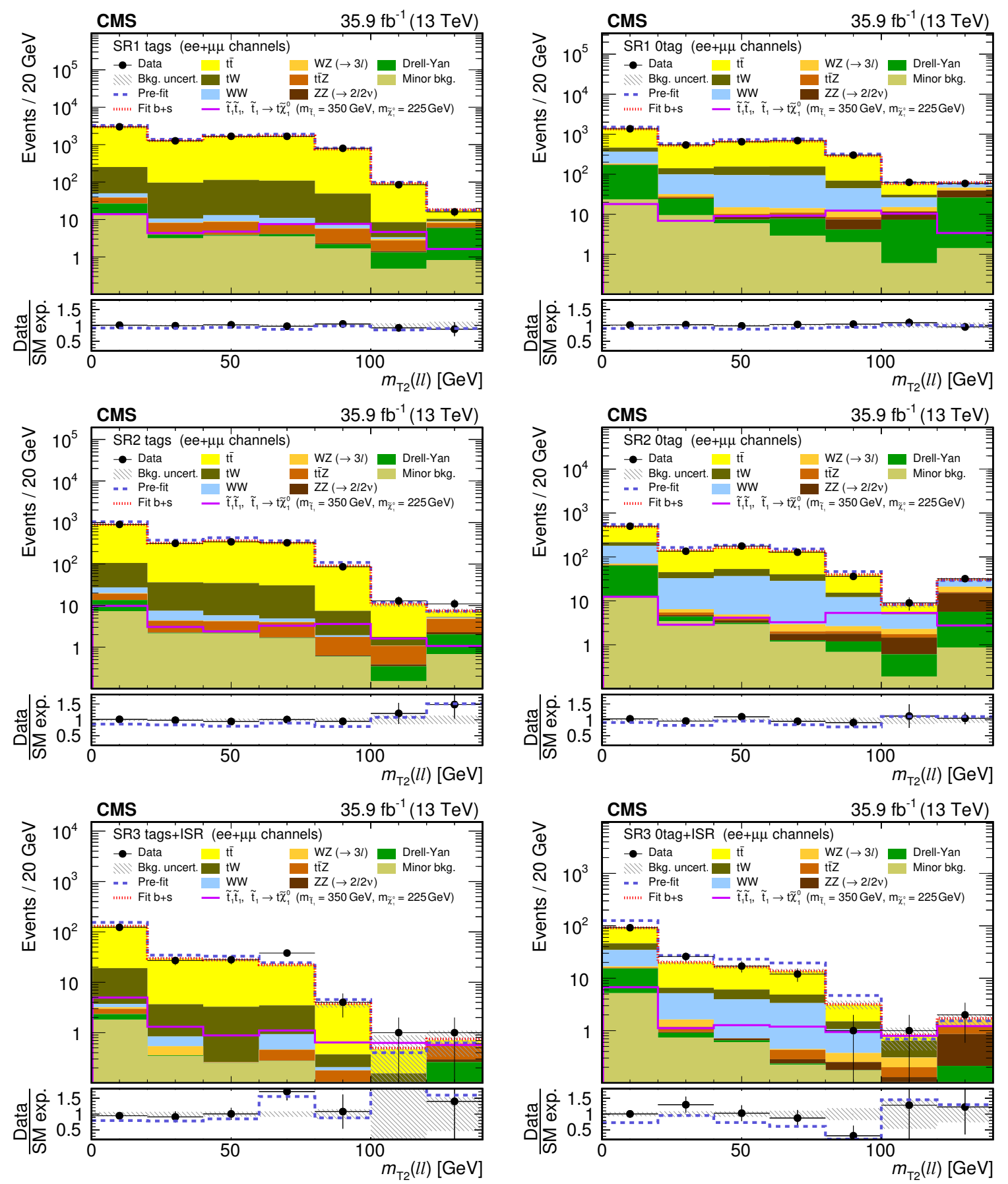

Figure 7. The same distributions of $m_{\mathrm{T} 2}(\ell \ell)$ as figure 6 , but for SF events. 


\begin{tabular}{|lcc|}
\hline \multirow{2}{*}{ Source of uncertainty } & \multicolumn{2}{c|}{ SM processes } \\
& Change in yields & Change in $m_{\mathrm{T} 2}(\ell \ell)$ shape \\
\hline Integrated luminosity & $2.5 \%$ & - \\
Trigger & $2 \%$ & - \\
Lepton ident./isolation & $4-5 \%$ & $<1 \%$ \\
Jet energy scale & $1-6 \%$ & $3-15 \%$ \\
Unclustered energy & $1-2 \%$ & $2-16 \%$ \\
b tagging & $<3 \%$ & $<2 \%$ \\
Renorm./fact. scales & $1-10 \%$ & $1-6 \%$ \\
PDFs & $1-5 \%$ & $2-8 \%$ \\
tĒZ normalization & $<1 \%$ & $<9 \%$ \\
WZ normalization & $<1 \%$ & $<1 \%$ \\
ZZ normalization & $<1 \%$ & $<5 \%$ \\
Drell-Yan normalization & $<4 \%$ & $1-11 \%$ \\
$m_{\mathrm{T} 2}(\ell \ell)$ shape (top quark) & - & $4-18 \%$ \\
$m_{\mathrm{T} 2}(\ell \ell)$ shape (WW) & - & $1-15 \%$ \\
$\mathrm{ZZ} K$ factors & - & $<3 \%$ \\
$m_{\mathrm{T} 2}(\ell \ell)$ shape (Drell-Yan) & - & $1-13 \%$ \\
Nonprompt leptons & $<1 \%$ & $<4 \%$ \\
$\mathrm{t} \overline{\mathrm{t}} p_{\mathrm{T}}$ reweighting & $1-4 \%$ & $1-8 \%$ \\
\hline & & \\
\hline
\end{tabular}

Table 5. Sizes of systematic uncertainties in the predicted yields for SM processes. The first column shows the range of the uncertainties in the global background normalization across the different SRs. The second column quantifies the effect on the $m_{\mathrm{T} 2}(\ell \ell)$ shape. This is computed by taking the maximum variation across the $m_{\mathrm{T} 2}(\ell \ell)$ bins (after renormalizing for the global change of all the distribution) in each SR. The range of this variation across the SRs is given.

upper limits with pp $\rightarrow \widetilde{\chi}_{1}^{+} \widetilde{\chi}_{1}^{-}$production cross sections, observed and expected exclusion regions in the $\left(m_{\widetilde{\chi}_{1}^{ \pm}}, m_{\widetilde{\chi}_{1}^{0}}\right)$ plane are also determined. Masses are excluded up to values of about 800 and $320 \mathrm{GeV}$ for the chargino and the neutralino, respectively. Limited sensitivity is found when the chargino is assumed to decay into a $\mathrm{W}$ boson and the lightest neutralino, due to the relatively small $\mathcal{B}$ for the leptonic decay of the $\mathrm{W}$ boson. For this scenario, we derive upper limits on chargino pair production cross section assuming a lightest neutralino mass of $1 \mathrm{GeV}$. Observed and expected upper limits as a function of the chargino mass are compared to theoretical cross sections in figure 8 (right).

Figure 9 shows the observed and expected 95\% CL upper limits on top squark production cross section for the two SMS considered. While the search strategy has been optimized for a compressed scenario, the results are presented on the whole $\left(m_{\widetilde{\mathfrak{t}}_{1}}, m_{\widetilde{\chi}_{1}^{0}}\right)$ plane for completeness. Also shown are the expected and observed exclusion regions when assuming NLO+NLL top squark pair production cross sections. When assuming the top 


\begin{tabular}{|c|c|c|c|c|}
\hline \multirow[t]{2}{*}{ Source of uncertainty } & \multicolumn{2}{|c|}{$\begin{array}{c}\tilde{\chi}_{1}^{ \pm} \rightarrow \widetilde{\ell} \nu(\ell \widetilde{\nu}) \rightarrow \ell \nu \widetilde{\chi}_{1}^{0} \\
\left(m_{\widetilde{\chi}_{1}^{ \pm}}=500 \mathrm{GeV}, m_{\widetilde{\chi}_{1}^{0}}=200 \mathrm{GeV}\right)\end{array}$} & \multicolumn{2}{|c|}{$\begin{array}{c}\widetilde{\mathrm{t}}_{1} \rightarrow \mathrm{t} \widetilde{\chi}_{1}^{0} \\
\left(m_{\widetilde{\mathrm{t}}_{1}}=350 \mathrm{GeV}, m_{\widetilde{\chi}_{1}^{0}}=225 \mathrm{GeV}\right)\end{array}$} \\
\hline & Yields & $m_{\mathrm{T} 2}(\ell \ell)$ shape & Yields & $m_{\mathrm{T} 2}(\ell \ell)$ shape \\
\hline Integrated luminosity & $2.5 \%$ & - & $2.5 \%$ & - \\
\hline Trigger & $2 \%$ & - & $2 \%$ & - \\
\hline Lepton ident./isolation & $4-5 \%$ & $<1 \%$ & $4-5 \%$ & $<1 \%$ \\
\hline Jet energy scale & $1-3 \%$ & $3-11 \%$ & $1-4 \%$ & $2-14 \%$ \\
\hline Unclustered energy & $1-2 \%$ & $8-13 \%$ & $1-2 \%$ & $2-7 \%$ \\
\hline $\mathrm{b}$ tagging & $<1 \%$ & $<1 \%$ & $1-3 \%$ & $<1 \%$ \\
\hline Renorm./fact. scales & $1-3 \%$ & $1-3 \%$ & $1-3 \%$ & $1-3 \%$ \\
\hline Lept. id./iso. (FASTSIM) & $4 \%$ & $<1 \%$ & $4 \%$ & $<1 \%$ \\
\hline b tagging (FASTSIM) & $<1 \%$ & $<1 \%$ & $<1 \%$ & $<1 \%$ \\
\hline$\vec{p}_{\mathrm{T}}^{\text {miss }}$ (FASTSIM) & $1-4 \%$ & $7-28 \%$ & $1-6 \%$ & $6-20 \%$ \\
\hline Pileup (FAStSim) & $1-6 \%$ & $4-9 \%$ & $2-4 \%$ & $2-14 \%$ \\
\hline ISR reweighting & $1-2 \%$ & $1-6 \%$ & $2-8 \%$ & $1-6 \%$ \\
\hline
\end{tabular}

Table 6. Same as in table 5 for two representative signal points, one for chargino pair production and one for top squark pair production.
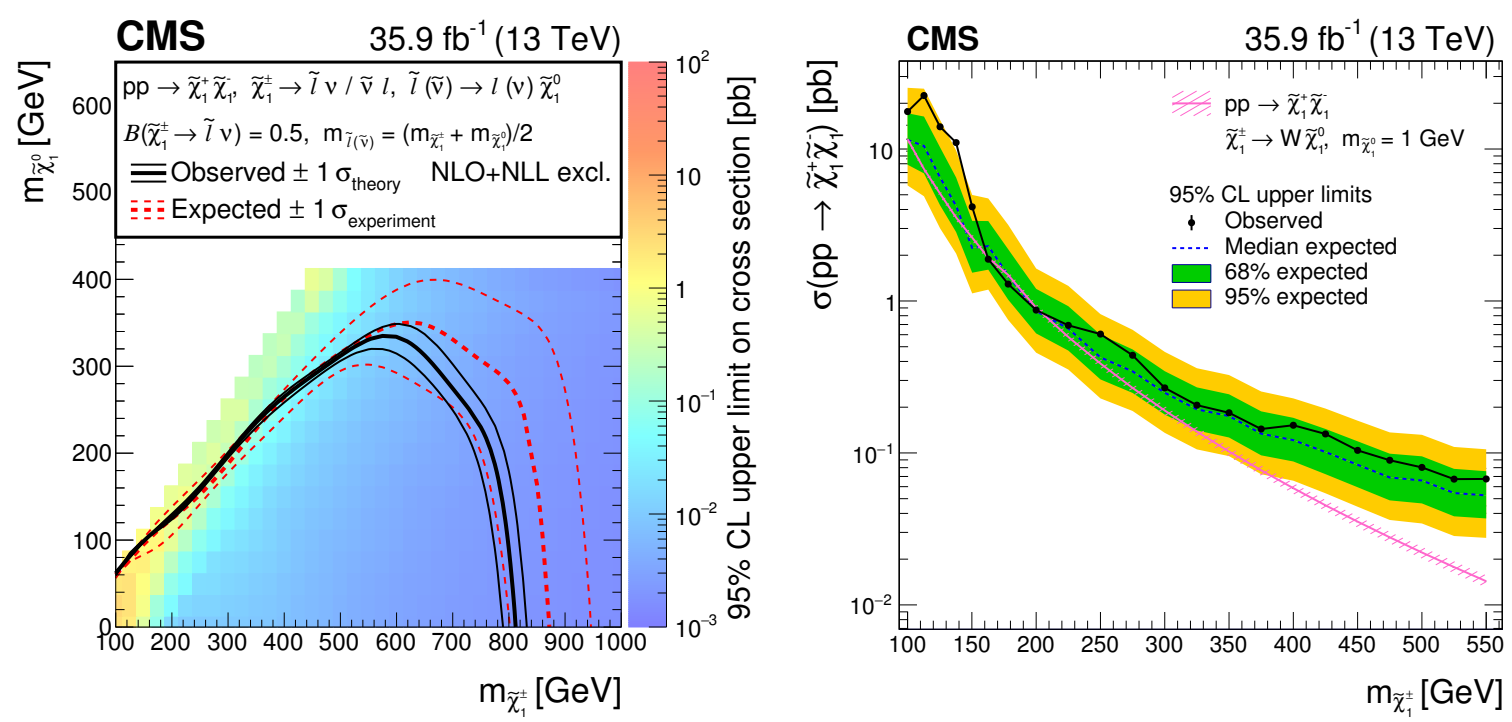

Figure 8. Left: upper limits at 95\% CL on chargino pair production cross section as a function of the chargino and neutralino masses, when the chargino undergoes a cascade decay $\widetilde{\chi}_{1}^{ \pm} \rightarrow \widetilde{\ell} \nu(\ell \widetilde{\nu}) \rightarrow$ $\ell \nu \widetilde{\chi}_{1}^{0}$. Exclusion regions in the plane $\left(m_{\widetilde{\chi}_{1}^{ \pm}}, m_{\widetilde{\chi}_{1}^{0}}\right)$ are determined by comparing the upper limits with the NLO+NLL production cross sections. The thick dashed red line shows the expected exclusion region. The thin dashed red lines show the variation of the exclusion regions due to the experimental uncertainties. The thick black line shows the observed exclusion region, while the thin black lines show the variation of the exclusion regions due to the theoretical uncertainties in the production cross section. Right: observed and expected upper limits at 95\% CL as a function of the chargino mass for a neutralino mass of $1 \mathrm{GeV}$, assuming chargino decays into a neutralino and a W boson $\left(\widetilde{\chi}_{1}^{ \pm} \rightarrow \mathrm{W} \widetilde{\chi}_{1}^{0}\right)$. 


\begin{tabular}{|c|c|c|c|c|c|c|c|c|}
\hline$m_{\mathrm{T} 2}(\ell \ell)$ & {$[\mathrm{GeV}]$} & $0-20$ & $20-40$ & $40-60$ & $60-80$ & $80-100$ & $100-120$ & $\geq 120$ \\
\hline \multicolumn{9}{|c|}{ DF events } \\
\hline \multirow{2}{*}{$\mathrm{SR} 1_{0 \text { tag }}^{\text {jets }}$} & Predicted & $1493 \pm 32$ & $558 \pm 12$ & $719 \pm 16$ & $730 \pm 16$ & $316 \pm 10$ & $45.1 \pm 3.1$ & $13.7 \pm 2.8$ \\
\hline & Observed & 1484 & 532 & 732 & 725 & 298 & 47 & 13 \\
\hline \multirow{2}{*}{$\mathrm{SR} 1_{\text {otag }}^{0 \text { jet }}$} & Predicted & $41.9 \pm 5$ & $27.4 \pm 3.8$ & $34.1 \pm 4.8$ & $42 \pm 5.5$ & $21.1 \pm 3.4$ & $6 \pm 1.3$ & $7.9 \pm 2.1$ \\
\hline & Observed & 39 & 24 & 33 & 44 & 13 & 6 & 9 \\
\hline \multirow{2}{*}{$\mathrm{SR} 2_{0 \text { tag }}^{\text {jets }}$} & Predicted & $534 \pm 15$ & $158.6 \pm 5.9$ & $167.9 \pm 6.1$ & $157.9 \pm 6.5$ & $42.4 \pm 2.9$ & $5.9 \pm 1$ & $9 \pm 1.7$ \\
\hline & Observed & 511 & 162 & 156 & 176 & 43 & 5 & 9 \\
\hline \multirow{2}{*}{$\mathrm{SR} 2_{\text {otag }}^{0 \text { jet }}$} & Predicted & $10.3 \pm 1.7$ & $7 \pm 1.5$ & $6.5 \pm 1.3$ & $6.9 \pm 1.3$ & $2.19 \pm 0.69$ & $1.59 \pm 0.7$ & $7.8 \pm 1.8$ \\
\hline & Observed & 10 & 4 & 4 & 6 & 2 & 2 & 7 \\
\hline \multirow{2}{*}{$\mathrm{SR} 3_{0 \text { tag }}$} & Predicted & $127.9 \pm 7.2$ & $28.3 \pm 2$ & $30.2 \pm 2.4$ & $23.1 \pm 2$ & $4.96 \pm 0.73$ & $1.12 \pm 0.38$ & $4.5 \pm 1.2$ \\
\hline & Observed & 116 & 35 & 29 & 21 & 3 & 1 & 5 \\
\hline \multicolumn{9}{|l|}{ SF events } \\
\hline \multirow{2}{*}{$\mathrm{SR} 1_{0 \text { tag }}^{\text {jets }}$} & Predicted & $1310 \pm 29$ & $499 \pm 12$ & $623 \pm 14$ & $634 \pm 15$ & $271.7 \pm 8.9$ & $51.6 \pm 3.5$ & $48.6 \pm 5.5$ \\
\hline & Observed & 1324 & 499 & 609 & 659 & 284 & 57 & 47 \\
\hline \multirow{2}{*}{$\mathrm{SR} 1_{0 \text { tag }}^{0 \text { jet }}$} & Predicted & $44.1 \pm 7.5$ & $28.5 \pm 4.1$ & $33.5 \pm 4.4$ & $33.5 \pm 4.5$ & $18.6 \pm 2.6$ & $7.7 \pm 1.6$ & $12.5 \pm 2.5$ \\
\hline & Observed & 43 & 40 & 39 & 33 & 17 & 6 & 12 \\
\hline \multirow{2}{*}{$\mathrm{SR} 2_{0 \text { tag }}^{\text {jets }}$} & Predicted & $474 \pm 14$ & $134.8 \pm 5.1$ & $155.1 \pm 5.5$ & $128.5 \pm 5.5$ & $37.1 \pm 2.5$ & $7.29 \pm 0.91$ & $23.9 \pm 2.4$ \\
\hline & Observed & 493 & 123 & 166 & 118 & 33 & 7 & 25 \\
\hline \multirow{2}{*}{$\mathrm{SR} 2_{0 \text { tag }}^{0 \text { jet }}$} & Predicted & $10.9 \pm 1.9$ & $7.8 \pm 1.8$ & $7.3 \pm 1.4$ & $7.9 \pm 1.3$ & $1.9 \pm 0.52$ & $1.28 \pm 0.58$ & $7.1 \pm 1.4$ \\
\hline & Observed & 8 & 12 & 11 & 10 & 3 & 2 & 7 \\
\hline \multirow{2}{*}{$\mathrm{SR} 3_{0 \operatorname{tag}}$} & Predicted & $112.8 \pm 6.3$ & $27.9 \pm 2.2$ & $24.2 \pm 1.8$ & $22.5 \pm 1.8$ & $5.2 \pm 1$ & $1.36 \pm 0.36$ & $10.6 \pm 1.2$ \\
\hline & Observed & 110 & 35 & 26 & 26 & 2 & 1 & 14 \\
\hline
\end{tabular}

Table 7. Observed and expected yields of DF (the upper half of table) and SF (the lower half) events in the SRs for the chargino search. The quoted uncertainties in the background predictions include statistical and systematic contributions.

squark to decay into a top quark and a neutralino, top squark (neutralino) masses are excluded up to about $420(360) \mathrm{GeV}$ in the compressed mass region where $\Delta m$ lies between the top quark and $\mathrm{W}$ boson masses. For the $\widetilde{\mathrm{t}}_{1} \rightarrow \mathrm{b} \widetilde{\chi}_{1}^{ \pm} \rightarrow \mathrm{bW} \widetilde{\chi}_{1}^{0}$ decay mode, a lower bound $\Delta m \approx 2 m_{\mathrm{W}}$ is set by the assumption that $m_{\widetilde{\chi}_{1}^{ \pm}}=\left(m_{\widetilde{\mathrm{t}}_{1}}+m_{\widetilde{\chi}_{1}^{0}}\right) / 2$. For $\Delta m \approx 2 m_{\mathrm{W}}$, top squark masses are excluded in the range $225-325 \mathrm{GeV}$. The uncovered region around a top squark mass of $200 \mathrm{GeV}$ in figure 9 (right) corresponds to a signal phase space similar to that of $t \bar{t}$ events, with little contribution from the neutralinos to $\vec{p}_{\mathrm{T}}^{\text {miss }}$. In this situation, the uncertainty in the modeling of $\vec{p}_{\mathrm{T}}^{\text {miss }}$ in FASTSim events becomes too large to provide any signal sensitivity.

\section{Summary}

A search has been presented for pair production of supersymmetric particles in events with two oppositely charged isolated leptons and missing transverse momentum. The data used consist of a sample of proton-proton collisions collected with the CMS detector during 


\begin{tabular}{|c|c|c|c|c|c|c|c|c|}
\hline$m_{\mathrm{T} 2}(\ell \ell)$ & {$[\mathrm{GeV}]$} & $0-20$ & $20-40$ & $40-60$ & $60-80$ & $80-100$ & $100-120$ & $\geq 120$ \\
\hline \multicolumn{9}{|c|}{ DF events } \\
\hline \multirow{2}{*}{$\mathrm{SR} 1_{\text {tags }}$} & Predicted & $3525 \pm 80$ & $1505 \pm 31$ & $1958 \pm 42$ & $2049 \pm 46$ & $897 \pm 22$ & $108.4 \pm 7.3$ & $13.4 \pm 2.2$ \\
\hline & Observed & 3534 & 1494 & 1938 & 2068 & 879 & 111 & 15 \\
\hline \multirow{2}{*}{$\mathrm{SR} 1_{0 \text { tag }}$} & Predicted & $1542 \pm 33$ & $588 \pm 13$ & $756 \pm 15$ & $771 \pm 19$ & $338.3 \pm 9.3$ & $50.6 \pm 3.8$ & $21 \pm 3.8$ \\
\hline & Observed & 1523 & 556 & 765 & 769 & 311 & 53 & 22 \\
\hline \multirow{2}{*}{$\mathrm{SR} 2_{\text {tags }}$} & Predicted & $1036 \pm 37$ & $363 \pm 13$ & $415 \pm 14$ & $377 \pm 14$ & $105.1 \pm 6.5$ & $12.3 \pm 2$ & $5.02 \pm 0.82$ \\
\hline & Observed & 1045 & 357 & 412 & 389 & 111 & 11 & 1 \\
\hline \multirow{2}{*}{$\mathrm{SR} 2_{0 \operatorname{tag}}$} & Predicted & $545 \pm 18$ & $164.3 \pm 7.3$ & $173.2 \pm 6.2$ & $165.1 \pm 6.8$ & $44.8 \pm 3.1$ & $7.1 \pm 1.4$ & $15.5 \pm 3$ \\
\hline & Observed & 521 & 166 & 160 & 182 & 45 & 7 & 16 \\
\hline \multirow{2}{*}{$\mathrm{SR} 3_{\text {tags }}^{\mathrm{ISR}}$} & Predicted & $152.1 \pm 9.9$ & $35.5 \pm 2.7$ & $32.3 \pm 2.3$ & $25 \pm 2.2$ & $4.67 \pm 0.77$ & $0.41 \pm 0.38$ & $0.41 \pm 0.26$ \\
\hline & Observed & 133 & 44 & 36 & 26 & 2 & 1 & 0 \\
\hline \multirow{2}{*}{$\mathrm{SR} 3_{0 \text { tag }}^{\mathrm{ISR}}$} & Predicted & $103.9 \pm 6.8$ & $21.3 \pm 1.9$ & $22.2 \pm 2.1$ & $15.4 \pm 1.6$ & $3.51 \pm 0.6$ & $0.53 \pm 0.21$ & $0.53 \pm 0.34$ \\
\hline & Observed & 100 & 27 & 22 & 12 & 3 & 0 & 1 \\
\hline \multicolumn{9}{|l|}{ SF events } \\
\hline \multirow{2}{*}{$\mathrm{SR} 1_{\text {tags }}$} & Predicted & $2979 \pm 68$ & $1277 \pm 30$ & $1644 \pm 35$ & $1712 \pm 37$ & $762 \pm 19$ & $91.9 \pm 6.1$ & $18.1 \pm 2.1$ \\
\hline & Observed & 3003 & 1266 & 1674 & 1671 & 798 & 85 & 16 \\
\hline \multirow{2}{*}{$\mathrm{SR} 1_{0 \operatorname{tag}}$} & Predicted & $1350 \pm 33$ & $526 \pm 13$ & $656 \pm 15$ & $670 \pm 17$ & $289.2 \pm 7.6$ & $57.9 \pm 4.2$ & $61.8 \pm 5.8$ \\
\hline & Observed & 1367 & 539 & 648 & 692 & 301 & 63 & 59 \\
\hline \multirow{2}{*}{$\mathrm{SR} 2_{\text {tags }}$} & Predicted & $888 \pm 30$ & $319 \pm 12$ & $363 \pm 14$ & $323 \pm 13$ & $90.5 \pm 5.5$ & $10.8 \pm 1.5$ & $7.43 \pm 0.98$ \\
\hline & Observed & 900 & 315 & 343 & 325 & 86 & 13 & 11 \\
\hline \multirow{2}{*}{$\mathrm{SR} 2_{0 \text { tag }}$} & Predicted & $487 \pm 16$ & $140.7 \pm 5.5$ & $161.9 \pm 5.9$ & $134.5 \pm 6.2$ & $39.6 \pm 2.7$ & $8.1 \pm 1.1$ & $30.6 \pm 3$ \\
\hline & Observed & 501 & 135 & 177 & 128 & 36 & 9 & 32 \\
\hline \multirow{2}{*}{$\mathrm{SR} 3_{\text {tags }}^{\mathrm{ISR}}$} & Predicted & $129.6 \pm 8.9$ & $29.6 \pm 2.1$ & $27.8 \pm 2.1$ & $22.2 \pm 1.9$ & $3.71 \pm 0.57$ & $0.47 \pm 0.42$ & $0.71 \pm 0.38$ \\
\hline & Observed & 123 & 27 & 28 & 38 & 4 & 1 & 1 \\
\hline \multirow{2}{*}{$\mathrm{SR} 3_{0 \text { tag }}^{\mathrm{ISR}}$} & Predicted & $91.5 \pm 6.1$ & $20.1 \pm 1.8$ & $16.5 \pm 1.4$ & $13.7 \pm 1.4$ & $3.14 \pm 0.58$ & $0.78 \pm 0.36$ & $1.63 \pm 0.42$ \\
\hline & Observed & 92 & 26 & 17 & 12 & 1 & 1 & 2 \\
\hline
\end{tabular}

Table 8. Observed and expected yields of DF (the upper half of table) and SF (the lower half) events in the SRs for the top squark search. The quoted uncertainties in the background predictions include statistical and systematic contributions.

the 2016 LHC run at a center-of-mass energy of $13 \mathrm{TeV}$, corresponding to an integrated luminosity of $35.9 \mathrm{fb}^{-1}$. No evidence for a deviation with respect to standard model predictions was observed in data. The results have been interpreted as upper limits on the cross sections of supersymmetric particle production for several simplified model spectra.

Chargino pair production has been investigated in two possible decay modes. If the chargino is assumed to undergo a cascade decay through sleptons, an exclusion region in the $\left(m_{\widetilde{\chi}_{1}^{ \pm}}, m_{\widetilde{\chi}_{1}^{0}}\right)$ plane can be derived, extending to chargino masses of $800 \mathrm{GeV}$ and neutralino masses of $320 \mathrm{GeV}$. These are the most stringent limits on this model to date. For chargino decays into a neutralino and a $\mathrm{W}$ boson, limits on the production cross section have been derived assuming a neutralino mass of $1 \mathrm{GeV}$, and chargino masses in the range 170-200 GeV have been excluded. 

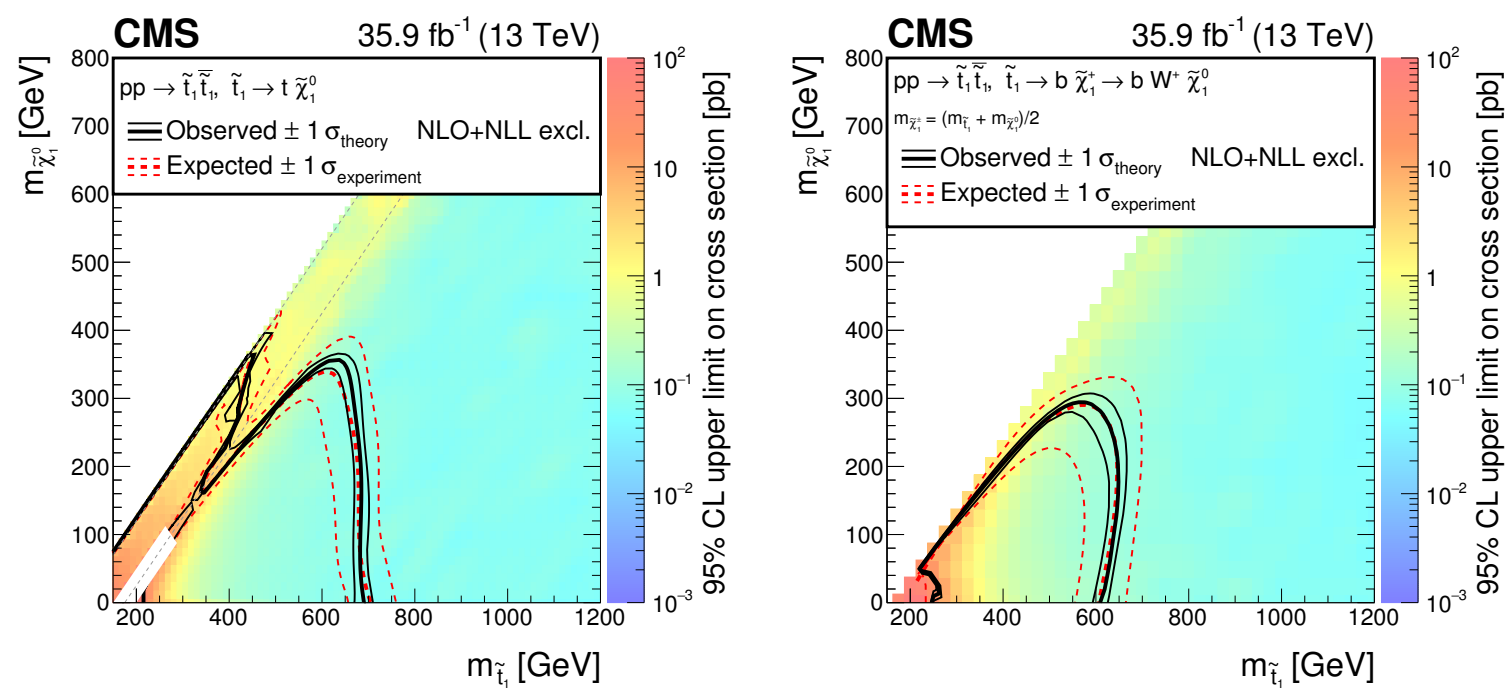

Figure 9. Upper limits at 95\% CL on top squark production cross section as a function of the top squark and neutralino masses. The plot on the left shows the results when top squark decays into a top quark and a neutralino are assumed. The two diagonal gray dashed lines enclose the compressed region where $m_{\mathrm{W}}<m_{\widetilde{\mathrm{t}}_{1}}-m_{\widetilde{\chi}_{1}^{0}} \lesssim m_{\mathrm{t}}$. The plot on the right gives the limits for top squarks decaying into a bottom quark and a chargino, with the latter successively decaying into a $\mathrm{W}$ boson and a neutralino. The mass of the chargino is assumed to be equal to the average of the top squark and neutralino masses. Exclusion regions in the plane $\left(m_{\widetilde{t}_{1}}, m_{\widetilde{\chi}_{1}^{0}}\right)$ are determined by comparing the upper limits with the NLO+NLL production cross sections. The thick dashed red line shows the expected exclusion region. The thin dashed red lines show the variation of the exclusion regions due to the experimental uncertainties. The thick black line shows the observed exclusion region, while the thin black lines show the variation of the exclusion regions due to the theoretical uncertainties in the production cross section.

Top squark pair production was also tested, with a focus on compressed decay modes. A model with the top squark decaying into a top quark and a neutralino was considered. In the region where $m_{\mathrm{W}}<m_{\widetilde{\mathrm{t}}_{1}}-m_{\widetilde{\chi}_{1}^{0}} \lesssim m_{\mathrm{t}}$, limits extend up to 420 and $360 \mathrm{GeV}$ for the top squark and neutralino masses, respectively. An alternative model has also been considered, where the top squark decays into a chargino and a bottom quark, with the chargino subsequently decaying into a $\mathrm{W}$ boson and the lightest neutralino. The mass of the chargino is assumed to be average between the top squark and neutralino masses, which gives a lower bound to the mass difference $(\Delta m)$ between the top squark and the neutralino of $\Delta m \approx 2 m_{\mathrm{W}}$. This search reduces by about $50 \mathrm{GeV}$ the minimum $\Delta m$ excluded in the previous result with two leptons in the final state [30] from the CMS Collaboration, excluding top squark masses in the range $225-325 \mathrm{GeV}$ for $\Delta m \approx 2 m_{\mathrm{W}}$.

In summary, by exploiting the full data set collected by the CMS experiment in 2016, this search extends the existing exclusion limits on the pair production of charginos decaying via sleptons [29], improving by about $70 \mathrm{GeV}$ the limit on the chargino mass for a massless neutralino. Exclusion limits on top squark pair production extend the results obtained by the CMS collaboration in final states with two oppositely charged leptons [30] to the compressed region, where they are competitive with the results obtained by the ATLAS Collaboration in the same decay channel [35]. 


\section{Acknowledgments}

We congratulate our colleagues in the CERN accelerator departments for the excellent performance of the LHC and thank the technical and administrative staffs at CERN and at other CMS institutes for their contributions to the success of the CMS effort. In addition, we gratefully acknowledge the computing centers and personnel of the Worldwide LHC Computing Grid for delivering so effectively the computing infrastructure essential to our analyses. Finally, we acknowledge the enduring support for the construction and operation of the LHC and the CMS detector provided by the following funding agencies: BMWFW and FWF (Austria); FNRS and FWO (Belgium); CNPq, CAPES, FAPERJ, and FAPESP (Brazil); MES (Bulgaria); CERN; CAS, MoST, and NSFC (China); COLCIENCIAS (Colombia); MSES and CSF (Croatia); RPF (Cyprus); SENESCYT (Ecuador); MoER, ERC IUT, and ERDF (Estonia); Academy of Finland, MEC, and HIP (Finland); CEA and CNRS/IN2P3 (France); BMBF, DFG, and HGF (Germany); GSRT (Greece); NKFIA (Hungary); DAE and DST (India); IPM (Iran); SFI (Ireland); INFN (Italy); MSIP and NRF (Republic of Korea); LAS (Lithuania); MOE and UM (Malaysia); BUAP, CINVESTAV, CONACYT, LNS, SEP, and UASLP-FAI (Mexico); MBIE (New Zealand); PAEC (Pakistan); MSHE and NSC (Poland); FCT (Portugal); JINR (Dubna); MON, RosAtom, RAS and RFBR (Russia); MESTD (Serbia); SEIDI, CPAN, PCTI and FEDER (Spain); Swiss Funding Agencies (Switzerland); MST (Taipei); ThEPCenter, IPST, STAR, and NSTDA (Thailand); TUBITAK and TAEK (Turkey); NASU and SFFR (Ukraine); STFC (United Kingdom); DOE and NSF (U.S.A.).

Individuals have received support from the Marie-Curie program and the European Research Council and Horizon 2020 Grant, contract No. 675440 (European Union); the Leventis Foundation; the A.P. Sloan Foundation; the Alexander von Humboldt Foundation; the Belgian Federal Science Policy Office; the Fonds pour la Formation à la Recherche dans l'Industrie et dans l'Agriculture (FRIA-Belgium); the Agentschap voor Innovatie door Wetenschap en Technologie (IWT-Belgium); the Ministry of Education, Youth and Sports (MEYS) of the Czech Republic; the Council of Scientific and Industrial Research, India; the HOMING PLUS program of the Foundation for Polish Science, cofinanced from European Union, Regional Development Fund, the Mobility Plus program of the Ministry of Science and Higher Education, the National Science Center (Poland), contracts Harmonia 2014/14/M/ST2/00428, Opus 2014/13/B/ST2/02543, 2014/15/B/ST2/03998, and 2015/19/B/ST2/02861, Sonata-bis 2012/07/E/ST2/01406; the National Priorities Research Program by Qatar National Research Fund; the Programa Severo Ochoa del Principado de Asturias; the Thalis and Aristeia programs cofinanced by EU-ESF and the Greek NSRF; the Rachadapisek Sompot Fund for Postdoctoral Fellowship, Chulalongkorn University and the Chulalongkorn Academic into Its 2nd Century Project Advancement Project (Thailand); the Welch Foundation, contract C-1845; and the Weston Havens Foundation (U.S.A.).

Open Access. This article is distributed under the terms of the Creative Commons Attribution License (CC-BY 4.0), which permits any use, distribution and reproduction in any medium, provided the original author(s) and source are credited. 


\section{References}

[1] E. Gildener and S. Weinberg, Symmetry Breaking and Scalar Bosons, Phys. Rev. D 13 (1976) 3333 [INSPIRE].

[2] G. 't Hooft, Naturalness, chiral symmetry, and spontaneous chiral symmetry breaking, NATO Sci. Ser. B 59 (1980) 135 [inSPIRE].

[3] G. Bertone, D. Hooper and J. Silk, Particle dark matter: Evidence, candidates and constraints, Phys. Rept. 405 (2005) 279 [hep-ph/0404175] [INSPIRE].

[4] J.L. Feng, Dark Matter Candidates from Particle Physics and Methods of Detection, Ann. Rev. Astron. Astrophys. 48 (2010) 495 [arXiv:1003.0904] [INSPIRE].

[5] T.A. Porter, R.P. Johnson and P.W. Graham, Dark Matter Searches with Astroparticle Data, Ann. Rev. Astron. Astrophys. 49 (2011) 155 [arXiv:1104.2836] [INSPIRE].

[6] P. Ramond, Dual Theory for Free Fermions, Phys. Rev. D 3 (1971) 2415 [InSPIRE].

[7] Y.A. Golfand and E.P. Likhtman, Extension of the Algebra of Poincaré Group Generators and Violation of p Invariance, JETP Lett. 13 (1971) 323 [INSPIRE].

[8] A. Neveu and J.H. Schwarz, Factorizable dual model of pions, Nucl. Phys. B 31 (1971) 86 [INSPIRE].

[9] D.V. Volkov and V.P. Akulov, Possible universal neutrino interaction, JETP Lett. 16 (1972) 438 [INSPIRE].

[10] J. Wess and B. Zumino, A Lagrangian Model Invariant Under Supergauge Transformations, Phys. Lett. B 49 (1974) 52 [inSPIRE].

[11] J. Wess and B. Zumino, Supergauge Transformations in Four-Dimensions, Nucl. Phys. B 70 (1974) 39 [INSPIRE].

[12] P. Fayet, Supergauge Invariant Extension of the Higgs Mechanism and a Model for the electron and Its Neutrino, Nucl. Phys. B 90 (1975) 104 [INSPIRE].

[13] H.P. Nilles, Supersymmetry, Supergravity and Particle Physics, Phys. Rept. 110 (1984) 1 [INSPIRE].

[14] S.P. Martin, A Supersymmetry primer, in Perspectives on Supersymmetry, Advanced Series on Directions in High Energy Physics, volume 21, World Scientific (2010) [hep-ph/9709356] [INSPIRE].

[15] E. Witten, Dynamical Breaking of Supersymmetry, Nucl. Phys. B 188 (1981) 513 [InSPIRE].

[16] S. Dimopoulos and H. Georgi, Softly Broken Supersymmetry and SU(5), Nucl. Phys. B 193 (1981) 150 [INSPIRE].

[17] R.K. Kaul and P. Majumdar, Cancellation of Quadratically Divergent Mass Corrections in Globally Supersymmetric Spontaneously Broken Gauge Theories, Nucl. Phys. B 199 (1982) 36 [INSPIRE].

[18] G.R. Farrar and P. Fayet, Phenomenology of the Production, Decay and Detection of New Hadronic States Associated with Supersymmetry, Phys. Lett. B 76 (1978) 575 [INSPIRE].

[19] CMS collaboration, The CMS Experiment at the CERN LHC, 2008 JINST 3 S08004 [INSPIRE].

[20] J. Alwall, P. Schuster and N. Toro, Simplified Models for a First Characterization of New Physics at the LHC, Phys. Rev. D 79 (2009) 075020 [arXiv:0810.3921] [INSPIRE]. 
[21] J. Alwall, M.-P. Le, M. Lisanti and J.G. Wacker, Model-Independent Jets plus Missing Energy Searches, Phys. Rev. D 79 (2009) 015005 [arXiv:0809.3264] [InSPIRE].

[22] LHC New Physics Working Group, D. Alves, Simplified Models for LHC New Physics Searches, J. Phys. G 39 (2012) 105005 [arXiv:1105.2838] [INSPIRE].

[23] CMS collaboration, Searches for electroweak production of charginos, neutralinos and sleptons decaying to leptons and $W, Z$ and Higgs bosons in pp collisions at 8 TeV, Eur. Phys. J. C 74 (2014) 3036 [arXiv:1405.7570] [INSPIRE].

[24] ATLAS collaboration, Search for direct production of charginos, neutralinos and sleptons in final states with two leptons and missing transverse momentum in pp collisions at $\sqrt{s}=8 \mathrm{TeV}$ with the ATLAS detector, JHEP 05 (2014) 071 [arXiv:1403.5294] [INSPIRE].

[25] ATLAS collaboration, Search for the direct production of charginos, neutralinos and staus in final states with at least two hadronically decaying taus and missing transverse momentum in pp collisions at $\sqrt{s}=8 \mathrm{TeV}$ with the ATLAS detector, JHEP 10 (2014) 096 [arXiv: 1407.0350] [INSPIRE].

[26] ATLAS collaboration, Search for the electroweak production of supersymmetric particles in $\sqrt{s}=8$ TeV pp collisions with the ATLAS detector, Phys. Rev. D 93 (2016) 052002 [arXiv: 1509.07152] [INSPIRE].

[27] ATLAS collaboration, Search for the direct production of charginos and neutralinos in final states with tau leptons in $\sqrt{s}=13 \mathrm{TeV}$ pp collisions with the ATLAS detector, Eur. Phys. J. C 78 (2018) 154 [arXiv: 1708.07875] [INSPIRE].

[28] ATLAS collaboration, Search for electroweak production of supersymmetric states in scenarios with compressed mass spectra at $\sqrt{s}=13 \mathrm{TeV}$ with the ATLAS detector, Phys. Rev. D 97 (2018) 052010 [arXiv:1712.08119] [INSPIRE].

[29] ATLAS collaboration, Search for electroweak production of supersymmetric particles in final states with two or three leptons at $\sqrt{s}=13 \mathrm{TeV}$ with the ATLAS detector, submitted to Eur. Phys. J. C (2018), arXiv: 1803.02762 [INSPIRE].

[30] CMS collaboration, Search for top squarks and dark matter particles in opposite-charge dilepton final states at $\sqrt{s}=13 \mathrm{TeV}$, Phys. Rev. D 97 (2018) 032009 [arXiv:1711.00752] [INSPIRE].

[31] CMS collaboration, Search for top squark pair production in pp collisions at $\sqrt{s}=13 \mathrm{TeV}$ using single lepton events, JHEP 10 (2017) 019 [arXiv:1706.04402] [INSPIRE].

[32] CMS collaboration, Search for direct production of supersymmetric partners of the top quark in the all-jets final state in proton-proton collisions at $\sqrt{s}=13 \mathrm{TeV}$, JHEP 10 (2017) 005 [arXiv: 1707.03316] [INSPIRE].

[33] ATLAS collaboration, Search for a scalar partner of the top quark in the jets plus missing transverse momentum final state at $\sqrt{s}=13 \mathrm{TeV}$ with the ATLAS detector, JHEP 12 (2017) 085 [arXiv: 1709.04183] [INSPIRE].

[34] ATLAS collaboration, Search for top-squark pair production in final states with one lepton, jets and missing transverse momentum using $36 \mathrm{fb}^{-1}$ of $\sqrt{s}=13 \mathrm{TeV}$ pp collision data with the ATLAS detector, JHEP 06 (2018) 108 [arXiv:1711.11520] [INSPIRE].

[35] ATLAS collaboration, Search for direct top squark pair production in final states with two leptons in $\sqrt{s}=13 \mathrm{TeV}$ pp collisions with the ATLAS detector, Eur. Phys. J. C 77 (2017) 898 [arXiv: 1708.03247] [INSPIRE]. 
[36] CMS collaboration, The CMS trigger system, 2017 JINST 12 P01020 [arXiv:1609.02366] [INSPIRE].

[37] P. Nason, A New method for combining NLO QCD with shower Monte Carlo algorithms, JHEP 11 (2004) 040 [hep-ph/0409146] [INSPIRE].

[38] S. Frixione, P. Nason and C. Oleari, Matching NLO QCD computations with Parton Shower simulations: the POWHEG method, JHEP 11 (2007) 070 [arXiv:0709.2092] [INSPIRE].

[39] S. Alioli, P. Nason, C. Oleari and E. Re, A general framework for implementing NLO calculations in shower Monte Carlo programs: the POWHEG BOX, JHEP 06 (2010) 043 [arXiv: 1002.2581] [INSPIRE].

[40] M. Czakon and A. Mitov, Top++: A Program for the Calculation of the Top-Pair Cross-Section at Hadron Colliders, Comput. Phys. Commun. 185 (2014) 2930 [arXiv:1112.5675] [INSPIRE].

[41] E. Re, Single-top Wt-channel production matched with parton showers using the POWHEG method, Eur. Phys. J. C 71 (2011) 1547 [arXiv: 1009. 2450] [INSPIRE].

[42] N. Kidonakis, NNLL threshold resummation for top-pair and single-top production, Phys. Part. Nucl. 45 (2014) 714 [arXiv:1210.7813] [INSPIRE].

[43] T. Melia, P. Nason, R. Röntsch and G. Zanderighi, $W^{+} W^{-}, W Z$ and $Z Z$ production in the POWHEG BOX, JHEP 11 (2011) 078 [arXiv:1107.5051] [INSPIRE].

[44] P. Nason and G. Zanderighi, $W^{+} W^{-}, W Z$ and $Z Z$ production in the POWHEG-BOX-V2, Eur. Phys. J. C 74 (2014) 2702 [arXiv:1311.1365] [INSPIRE].

[45] T. Gehrmann et al., $W^{+} W^{-}$Production at Hadron Colliders in Next to Next to Leading Order QCD, Phys. Rev. Lett. 113 (2014) 212001 [arXiv:1408.5243] [INSPIRE].

[46] CMS collaboration, Measurements of properties of the Higgs boson decaying into the four-lepton final state in pp collisions at $\sqrt{s}=13 \mathrm{TeV}$, JHEP 11 (2017) 047 [arXiv: 1706.09936] [INSPIRE].

[47] J.M. Campbell and R.K. Ellis, MCFM for the Tevatron and the LHC, Nucl. Phys. Proc. Suppl. 205-206 (2010) 10 [arXiv: 1007.3492] [INSPIRE].

[48] F. Caola, K. Melnikov, R. Röntsch and L. Tancredi, QCD corrections to $W^{+} W^{-}$production through gluon fusion, Phys. Lett. B 754 (2016) 275 [arXiv:1511.08617] [INSPIRE].

[49] J. Alwall et al., The automated computation of tree-level and next-to-leading order differential cross sections and their matching to parton shower simulations, JHEP 07 (2014) 079 [arXiv: 1405.0301] [INSPIRE].

[50] R. Gavin, Y. Li, F. Petriello and S. Quackenbush, FEWZ 2.0: A code for hadronic Z production at next-to-next-to-leading order, Comput. Phys. Commun. 182 (2011) 2388 [arXiv: 1011.3540] [INSPIRE].

[51] M.V. Garzelli, A. Kardos, C.G. Papadopoulos and Z. Trócsányi, $t \bar{t} W^{ \pm}$and $t \bar{t} Z$ Hadroproduction at NLO accuracy in QCD with Parton Shower and Hadronization effects, JHEP 11 (2012) 056 [arXiv:1208.2665] [INSPIRE].

[52] LHC Higgs Cross Section Working Group, J.R. Andersen et al., Handbook of LHC Higgs Cross Sections: 3. Higgs Properties, CERN-2013-004 (2013) [FERMILAB-CONF-13-667-T] [arXiv: 1307.1347] [INSPIRE]. 
[53] W. Beenakker, M. Klasen, M. Krämer, T. Plehn, M. Spira and P.M. Zerwas, The Production of charginos/neutralinos and sleptons at hadron colliders, Phys. Rev. Lett. 83 (1999) 3780 [Erratum ibid. 100 (2008) 029901] [hep-ph/9906298] [INSPIRE].

[54] B. Fuks, M. Klasen, D.R. Lamprea and M. Rothering, Gaugino production in proton-proton collisions at a center-of-mass energy of 8 TeV, JHEP 10 (2012) 081 [arXiv:1207.2159] [INSPIRE].

[55] B. Fuks, M. Klasen, D.R. Lamprea and M. Rothering, Precision predictions for electroweak superpartner production at hadron colliders with Resummino, Eur. Phys. J. C 73 (2013) 2480 [arXiv: 1304.0790] [INSPIRE].

[56] W. Beenakker, R. Hopker, M. Spira and P.M. Zerwas, Squark and gluino production at hadron colliders, Nucl. Phys. B 492 (1997) 51 [hep-ph/9610490] [INSPIRE].

[57] A. Kulesza and L. Motyka, Threshold resummation for squark-antisquark and gluino-pair production at the LHC, Phys. Rev. Lett. 102 (2009) 111802 [arXiv:0807.2405] [INSPIRE].

[58] A. Kulesza and L. Motyka, Soft gluon resummation for the production of gluino-gluino and squark-antisquark pairs at the LHC, Phys. Rev. D 80 (2009) 095004 [arXiv:0905.4749] [INSPIRE].

[59] W. Beenakker, S. Brensing, M. Krämer, A. Kulesza, E. Laenen and I. Niessen, Soft-gluon resummation for squark and gluino hadroproduction, JHEP 12 (2009) 041 [arXiv: 0909.4418] [INSPIRE].

[60] W. Beenakker et al., Squark and Gluino Hadroproduction, Int. J. Mod. Phys. A 26 (2011) 2637 [arXiv: 1105.1110] [INSPIRE].

[61] C. Borschensky et al., Squark and gluino production cross sections in pp collisions at $\sqrt{s}=13,14,33$ and $100 \mathrm{TeV}$, Eur. Phys. J. C 74 (2014) 3174 [arXiv:1407.5066] [inSPIRE].

[62] NNPDF collaboration, R.D. Ball et al., Parton distributions for the LHC Run II, JHEP 04 (2015) 040 [arXiv: 1410.8849] [InSPIRE].

[63] T. Sjöstrand et al., An Introduction to PYTHIA 8.2, Comput. Phys. Commun. 191 (2015) 159 [arXiv: 1410.3012] [inSPIRE].

[64] CMS collaboration, Event generator tunes obtained from underlying event and multiparton scattering measurements, Eur. Phys. J. C 76 (2016) 155 [arXiv:1512.00815] [INSPIRE].

[65] CMS collaboration, Investigations of the impact of the parton shower tuning in PYTHIA 8 in the modelling of $t \bar{t}$ at $\sqrt{s}=8$ and $13 \mathrm{TeV}$, CMS-PAS-TOP-16-021 (2016) [INSPIRE].

[66] A. Kalogeropoulos and J. Alwall, The SysCalc code: A tool to derive theoretical systematic uncertainties, arXiv: 1801.08401 [INSPIRE].

[67] GEANT4 collaboration, S. Agostinelli et al., GEANT4: A Simulation toolkit, Nucl. Instrum. Meth. A 506 (2003) 250 [INSPIRE].

[68] CMS collaboration, The fast simulation of the CMS detector at LHC, J. Phys. Conf. Ser. 331 (2011) 032049 [INSPIRE].

[69] CMS collaboration, Search for top-squark pair production in the single-lepton final state in pp collisions at $\sqrt{s}=8 \mathrm{TeV}$, Eur. Phys. J. C 73 (2013) 2677 [arXiv:1308.1586] [INSPIRE].

[70] CMS collaboration, Particle-flow reconstruction and global event description with the CMS detector, 2017 JINST 12 P10003 [arXiv:1706. 04965] [INSPIRE]. 
[71] M. Cacciari, G.P. Salam and G. Soyez, The anti- $k_{t}$ jet clustering algorithm, JHEP 04 (2008) 063 [arXiv:0802.1189] [INSPIRE].

[72] M. Cacciari, G.P. Salam and G. Soyez, FastJet User Manual, Eur. Phys. J. C 72 (2012) 1896 [arXiv: 1111.6097] [INSPIRE].

[73] CMS collaboration, Performance of CMS muon reconstruction in pp collision events at $\sqrt{s}=7 \mathrm{TeV}, 2012$ JINST 7 P10002 [arXiv:1206.4071] [INSPIRE].

[74] CMS collaboration, Performance of Electron Reconstruction and Selection with the CMS Detector in Proton-Proton Collisions at $\sqrt{s}=8$ TeV, 2015 JINST $10 \mathrm{P} 06005$ [arXiv: 1502.02701] [INSPIRE].

[75] CMS collaboration, Jet energy scale and resolution in the CMS experiment in pp collisions at $8 \mathrm{TeV}, 2017$ JINST 12 P02014 [arXiv: 1607.03663] [INSPIRE].

[76] CMS collaboration, Identification of heavy-flavour jets with the CMS detector in pp collisions at $13 \mathrm{TeV}, 2018$ JINST $13 \mathrm{P} 05011$ [arXiv:1712.07158] [INSPIRE].

[77] CMS collaboration, Performance of the CMS missing transverse momentum reconstruction in pp data at $\sqrt{s}=8 \mathrm{TeV}, 2015$ JINST $10 \mathrm{P} 02006$ [arXiv:1411.0511] [INSPIRE].

[78] C.G. Lester and D.J. Summers, Measuring masses of semiinvisibly decaying particles pair produced at hadron colliders, Phys. Lett. B 463 (1999) 99 [hep-ph/9906349] [INSPIRE].

[79] CMS collaboration, CMS Luminosity Measurements for the 2016 Data Taking Period, CMS-PAS-LUM-17-001 (2017) [INSPIRE].

[80] CMS collaboration, Measurement of differential cross sections for top quark pair production using the lepton + jets final state in proton-proton collisions at $13 \mathrm{TeV}$, Phys. Rev. D 95 (2017) 092001 [arXiv: 1610.04191] [INSPIRE].

[81] CMS collaboration, Measurement of the differential cross section for top quark pair production in pp collisions at $\sqrt{s}=8 \mathrm{TeV}$, Eur. Phys. J. C 75 (2015) 542 [arXiv: 1505.04480] [INSPIRE].

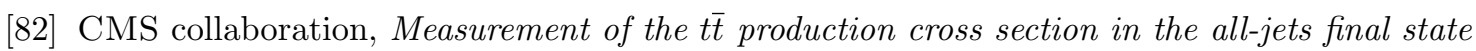
in pp collisions at $\sqrt{s}=8 \mathrm{TeV}$, Eur. Phys. J. C 76 (2016) 128 [arXiv:1509.06076] [INSPIRE].

[83] T. Junk, Confidence level computation for combining searches with small statistics, Nucl. Instrum. Meth. A 434 (1999) 435 [hep-ex/9902006] [INSPIRE].

[84] A.L. Read, Presentation of search results: The CLs technique, J. Phys. G 28 (2002) 2693 [INSPIRE].

[85] G. Cowan, K. Cranmer, E. Gross and O. Vitells, Asymptotic formulae for likelihood-based tests of new physics, Eur. Phys. J. C 71 (2011) 1554 [Erratum ibid. C 73 (2013) 2501] [arXiv: 1007.1727] [INSPIRE]. 


\section{The CMS collaboration}

\section{Yerevan Physics Institute, Yerevan, Armenia}

A.M. Sirunyan, A. Tumasyan

\section{Institut für Hochenergiephysik, Wien, Austria}

W. Adam, F. Ambrogi, E. Asilar, T. Bergauer, J. Brandstetter, M. Dragicevic, J. Erö, A. Escalante Del Valle, M. Flechl, R. Frühwirth ${ }^{1}$, V.M. Ghete, J. Hrubec, M. Jeitler ${ }^{1}$, N. Krammer, I. Krätschmer, D. Liko, T. Madlener, I. Mikulec, N. Rad, H. Rohringer, J. Schieck ${ }^{1}$, R. Schöfbeck, M. Spanring, D. Spitzbart, A. Taurok, W. Waltenberger, J. Wittmann, C.-E. Wulz ${ }^{1}$, M. Zarucki

\section{Institute for Nuclear Problems, Minsk, Belarus}

V. Chekhovsky, V. Mossolov, J. Suarez Gonzalez

\section{Universiteit Antwerpen, Antwerpen, Belgium}

E.A. De Wolf, D. Di Croce, X. Janssen, J. Lauwers, M. Pieters, M. Van De Klundert, H. Van Haevermaet, P. Van Mechelen, N. Van Remortel

\section{Vrije Universiteit Brussel, Brussel, Belgium}

S. Abu Zeid, F. Blekman, J. D'Hondt, I. De Bruyn, J. De Clercq, K. Deroover, G. Flouris, D. Lontkovskyi, S. Lowette, I. Marchesini, S. Moortgat, L. Moreels, Q. Python, K. Skovpen, S. Tavernier, W. Van Doninck, P. Van Mulders, I. Van Parijs

\section{Université Libre de Bruxelles, Bruxelles, Belgium}

D. Beghin, B. Bilin, H. Brun, B. Clerbaux, G. De Lentdecker, H. Delannoy, B. Dorney, G. Fasanella, L. Favart, R. Goldouzian, A. Grebenyuk, A.K. Kalsi, T. Lenzi, J. Luetic, N. Postiau, E. Starling, L. Thomas, C. Vander Velde, P. Vanlaer, D. Vannerom, Q. Wang

\section{Ghent University, Ghent, Belgium}

T. Cornelis, D. Dobur, A. Fagot, M. Gul, I. Khvastunov², D. Poyraz, C. Roskas, D. Trocino, M. Tytgat, W. Verbeke, B. Vermassen, M. Vit, N. Zaganidis

\section{Université Catholique de Louvain, Louvain-la-Neuve, Belgium}

H. Bakhshiansohi, O. Bondu, S. Brochet, G. Bruno, C. Caputo, P. David, C. Delaere, M. Delcourt, B. Francois, A. Giammanco, G. Krintiras, V. Lemaitre, A. Magitteri, A. Mertens, M. Musich, K. Piotrzkowski, A. Saggio, M. Vidal Marono, S. Wertz, J. Zobec

\section{Centro Brasileiro de Pesquisas Fisicas, Rio de Janeiro, Brazil}

F.L. Alves, G.A. Alves, L. Brito, M. Correa Martins Junior, G. Correia Silva, C. Hensel, A. Moraes, M.E. Pol, P. Rebello Teles

\section{Universidade do Estado do Rio de Janeiro, Rio de Janeiro, Brazil}

E. Belchior Batista Das Chagas, W. Carvalho, J. Chinellato ${ }^{3}$, E. Coelho, E.M. Da Costa, G.G. Da Silveira ${ }^{4}$, D. De Jesus Damiao, C. De Oliveira Martins, S. Fonseca De Souza, H. Malbouisson, D. Matos Figueiredo, M. Melo De Almeida, C. Mora Herrera, L. Mundim, H. Nogima, W.L. Prado Da Silva, L.J. Sanchez Rosas, A. Santoro, A. Sznajder, M. Thiel, E.J. Tonelli Manganote ${ }^{3}$, F. Torres Da Silva De Araujo, A. Vilela Pereira 
Universidade Estadual Paulista ${ }^{a}$, Universidade Federal do ABC ${ }^{b}$, São Paulo, Brazil

S. Ahuja ${ }^{a}$, C.A. Bernardes ${ }^{a}$, L. Calligaris $^{a}$, T.R. Fernandez Perez Tomei ${ }^{a}$, E.M. Gregores $^{b}{ }$ P.G. Mercadante ${ }^{b}$, S.F. Novaes ${ }^{a}$, SandraS. Padula ${ }^{a}$, D. Romero Abad $^{b}$

Institute for Nuclear Research and Nuclear Energy, Bulgarian Academy of Sciences, Sofia, Bulgaria

A. Aleksandrov, R. Hadjiiska, P. Iaydjiev, A. Marinov, M. Misheva, M. Rodozov,

M. Shopova, G. Sultanov

University of Sofia, Sofia, Bulgaria

A. Dimitrov, L. Litov, B. Pavlov, P. Petkov

Beihang University, Beijing, China

W. Fang ${ }^{5}$, X. Gao ${ }^{5}$, L. Yuan

Institute of High Energy Physics, Beijing, China

M. Ahmad, J.G. Bian, G.M. Chen, H.S. Chen, M. Chen, Y. Chen, C.H. Jiang, D. Leggat, H. Liao, Z. Liu, F. Romeo, S.M. Shaheen ${ }^{6}$, A. Spiezia, J. Tao, C. Wang, Z. Wang, E. Yazgan,

H. Zhang, J. Zhao

State Key Laboratory of Nuclear Physics and Technology, Peking University, Beijing, China

Y. Ban, G. Chen, A. Levin, J. Li, L. Li, Q. Li, Y. Mao, S.J. Qian, D. Wang, Z. Xu

Tsinghua University, Beijing, China

Y. Wang

Universidad de Los Andes, Bogota, Colombia

C. Avila, A. Cabrera, C.A. Carrillo Montoya, L.F. Chaparro Sierra, C. Florez, C.F. González Hernández, M.A. Segura Delgado

University of Split, Faculty of Electrical Engineering, Mechanical Engineering and Naval Architecture, Split, Croatia

B. Courbon, N. Godinovic, D. Lelas, I. Puljak, T. Sculac

University of Split, Faculty of Science, Split, Croatia

Z. Antunovic, M. Kovac

Institute Rudjer Boskovic, Zagreb, Croatia

V. Brigljevic, D. Ferencek, K. Kadija, B. Mesic, A. Starodumov ${ }^{7}$, T. Susa

University of Cyprus, Nicosia, Cyprus

M.W. Ather, A. Attikis, M. Kolosova, G. Mavromanolakis, J. Mousa, C. Nicolaou, F. Ptochos, P.A. Razis, H. Rykaczewski

Charles University, Prague, Czech Republic

M. Finger ${ }^{8}$, M. Finger Jr. ${ }^{8}$ 
Escuela Politecnica Nacional, Quito, Ecuador

E. Ayala

Universidad San Francisco de Quito, Quito, Ecuador

E. Carrera Jarrin

Academy of Scientific Research and Technology of the Arab Republic of Egypt, Egyptian Network of High Energy Physics, Cairo, Egypt

Y. Assran ${ }^{9,10}$, S. Khalil ${ }^{11}$, A. Mahrous ${ }^{12}$

National Institute of Chemical Physics and Biophysics, Tallinn, Estonia

S. Bhowmik, A. Carvalho Antunes De Oliveira, R.K. Dewanjee, K. Ehataht, M. Kadastik, M. Raidal, C. Veelken

Department of Physics, University of Helsinki, Helsinki, Finland

P. Eerola, H. Kirschenmann, J. Pekkanen, M. Voutilainen

Helsinki Institute of Physics, Helsinki, Finland

J. Havukainen, J.K. Heikkilä, T. Järvinen, V. Karimäki, R. Kinnunen, T. Lampén, K. Lassila-Perini, S. Laurila, S. Lehti, T. Lindén, P. Luukka, T. Mäenpää, H. Siikonen, E. Tuominen, J. Tuominiemi

\section{Lappeenranta University of Technology, Lappeenranta, Finland}

T. Tuuva

IRFU, CEA, Université Paris-Saclay, Gif-sur-Yvette, France

M. Besancon, F. Couderc, M. Dejardin, D. Denegri, J.L. Faure, F. Ferri, S. Ganjour, A. Givernaud, P. Gras, G. Hamel de Monchenault, P. Jarry, C. Leloup, E. Locci, J. Malcles, G. Negro, J. Rander, A. Rosowsky, M.Ö. Sahin, M. Titov

Laboratoire Leprince-Ringuet, Ecole polytechnique, CNRS/IN2P3, Université Paris-Saclay, Palaiseau, France

A. Abdulsalam ${ }^{13}$, C. Amendola, I. Antropov, F. Beaudette, P. Busson, C. Charlot, R. Granier de Cassagnac, I. Kucher, A. Lobanov, J. Martin Blanco, M. Nguyen, C. Ochando, G. Ortona, P. Paganini, P. Pigard, R. Salerno, J.B. Sauvan, Y. Sirois, A.G. Stahl Leiton, A. Zabi, A. Zghiche

Université de Strasbourg, CNRS, IPHC UMR 7178, Strasbourg, France J.-L. Agram ${ }^{14}$, J. Andrea, D. Bloch, J.-M. Brom, E.C. Chabert, V. Cherepanov, C. Collard, E. Conte ${ }^{14}$, J.-C. Fontaine ${ }^{14}$, D. Gelé, U. Goerlach, M. Jansová, A.-C. Le Bihan, N. Tonon, P. Van Hove

Centre de Calcul de l'Institut National de Physique Nucleaire et de Physique des Particules, CNRS/IN2P3, Villeurbanne, France

S. Gadrat 
Université de Lyon, Université Claude Bernard Lyon 1, CNRS-IN2P3, Institut de Physique Nucléaire de Lyon, Villeurbanne, France

S. Beauceron, C. Bernet, G. Boudoul, N. Chanon, R. Chierici, D. Contardo, P. Depasse, H. El Mamouni, J. Fay, L. Finco, S. Gascon, M. Gouzevitch, G. Grenier, B. Ille, F. Lagarde, I.B. Laktineh, H. Lattaud, M. Lethuillier, L. Mirabito, A.L. Pequegnot, S. Perries, A. Popov ${ }^{15}$, V. Sordini, M. Vander Donckt, S. Viret, S. Zhang

\section{Georgian Technical University, Tbilisi, Georgia}

A. Khvedelidze ${ }^{8}$

Tbilisi State University, Tbilisi, Georgia

Z. Tsamalaidze ${ }^{8}$

RWTH Aachen University, I. Physikalisches Institut, Aachen, Germany

C. Autermann, L. Feld, M.K. Kiesel, K. Klein, M. Lipinski, M. Preuten, M.P. Rauch, C. Schomakers, J. Schulz, M. Teroerde, B. Wittmer, V. Zhukov ${ }^{15}$

RWTH Aachen University, III. Physikalisches Institut A, Aachen, Germany

A. Albert, D. Duchardt, M. Endres, M. Erdmann, T. Esch, R. Fischer, S. Ghosh, A. Güth, T. Hebbeker, C. Heidemann, K. Hoepfner, H. Keller, S. Knutzen, L. Mastrolorenzo, M. Merschmeyer, A. Meyer, P. Millet, S. Mukherjee, T. Pook, M. Radziej, H. Reithler, M. Rieger, F. Scheuch, A. Schmidt, D. Teyssier

RWTH Aachen University, III. Physikalisches Institut B, Aachen, Germany

G. Flügge, O. Hlushchenko, B. Kargoll, T. Kress, A. Künsken, T. Müller, A. Nehrkorn, A. Nowack, C. Pistone, O. Pooth, H. Sert, A. Stahl ${ }^{16}$

\section{Deutsches Elektronen-Synchrotron, Hamburg, Germany}

M. Aldaya Martin, T. Arndt, C. Asawatangtrakuldee, I. Babounikau, K. Beernaert, O. Behnke, U. Behrens, A. Bermúdez Martínez, D. Bertsche, A.A. Bin Anuar, K. Borras ${ }^{17}$, V. Botta, A. Campbell, P. Connor, C. Contreras-Campana, F. Costanza, V. Danilov, A. De Wit, M.M. Defranchis, C. Diez Pardos, D. Domínguez Damiani, G. Eckerlin, T. Eichhorn, A. Elwood, E. Eren, E. Gallo ${ }^{18}$, A. Geiser, J.M. Grados Luyando, A. Grohsjean, P. Gunnellini, M. Guthoff, M. Haranko, A. Harb, J. Hauk, H. Jung, M. Kasemann, J. Keaveney, C. Kleinwort, J. Knolle, D. Krücker, W. Lange, A. Lelek, T. Lenz, K. Lipka, W. Lohmann ${ }^{19}$, R. Mankel, I.-A. Melzer-Pellmann, A.B. Meyer, M. Meyer, M. Missiroli, G. Mittag, J. Mnich, V. Myronenko, S.K. Pflitsch, D. Pitzl, A. Raspereza, M. Savitskyi, P. Saxena, P. Schütze, C. Schwanenberger, R. Shevchenko, A. Singh, N. Stefaniuk, H. Tholen, O. Turkot, A. Vagnerini, G.P. Van Onsem, R. Walsh, Y. Wen, K. Wichmann, C. Wissing, O. Zenaiev

\section{University of Hamburg, Hamburg, Germany}

R. Aggleton, S. Bein, L. Benato, A. Benecke, V. Blobel, M. Centis Vignali, T. Dreyer, E. Garutti, D. Gonzalez, J. Haller, A. Hinzmann, A. Karavdina, G. Kasieczka, R. Klanner, R. Kogler, N. Kovalchuk, S. Kurz, V. Kutzner, J. Lange, D. Marconi, J. Multhaup, M. Niedziela, D. Nowatschin, A. Perieanu, A. Reimers, O. Rieger, C. Scharf, P. Schleper, 
S. Schumann, J. Schwandt, J. Sonneveld, H. Stadie, G. Steinbrück, F.M. Stober, M. Stöver, D. Troendle, A. Vanhoefer, B. Vormwald

Karlsruher Institut fuer Technology

M. Akbiyik, C. Barth, M. Baselga, S. Baur, E. Butz, R. Caspart, T. Chwalek, F. Colombo, W. De Boer, A. Dierlamm, K. El Morabit, N. Faltermann, B. Freund, M. Giffels, M.A. Harrendorf, F. Hartmann ${ }^{16}$, S.M. Heindl, U. Husemann, F. Kassel ${ }^{16}$, I. Katkov ${ }^{15}$, S. Kudella, H. Mildner, S. Mitra, M.U. Mozer, Th. Müller, M. Plagge, G. Quast, K. Rabbertz, M. Schröder, I. Shvetsov, G. Sieber, H.J. Simonis, R. Ulrich, S. Wayand, M. Weber, T. Weiler, S. Williamson, C. Wöhrmann, R. Wolf

Institute of Nuclear and Particle Physics (INPP), NCSR Demokritos, Aghia Paraskevi, Greece

G. Anagnostou, G. Daskalakis, T. Geralis, A. Kyriakis, D. Loukas, G. Paspalaki, I. TopsisGiotis

National and Kapodistrian University of Athens, Athens, Greece

G. Karathanasis, S. Kesisoglou, P. Kontaxakis, A. Panagiotou, N. Saoulidou, E. Tziaferi, K. Vellidis

National Technical University of Athens, Athens, Greece

K. Kousouris, I. Papakrivopoulos, G. Tsipolitis

University of Ioánnina, Ioánnina, Greece

I. Evangelou, C. Foudas, P. Gianneios, P. Katsoulis, P. Kokkas, S. Mallios, N. Manthos, I. Papadopoulos, E. Paradas, J. Strologas, F.A. Triantis, D. Tsitsonis

MTA-ELTE Lendület CMS Particle and Nuclear Physics Group, Eötvös Loránd University, Budapest, Hungary

M. Bartók ${ }^{20}$, M. Csanad, N. Filipovic, P. Major, M.I. Nagy, G. Pasztor, O. Surányi, G.I. Veres

Wigner Research Centre for Physics, Budapest, Hungary

G. Bencze, C. Hajdu, D. Horvath ${ }^{21}$, Á. Hunyadi, F. Sikler, T.Á. Vámi, V. Veszpremi, G. Vesztergombi ${ }^{\dagger}$

Institute of Nuclear Research ATOMKI, Debrecen, Hungary

N. Beni, S. Czellar, J. Karancsi ${ }^{22}$, A. Makovec, J. Molnar, Z. Szillasi

Institute of Physics, University of Debrecen, Debrecen, Hungary

P. Raics, Z.L. Trocsanyi, B. Ujvari

Indian Institute of Science (IISc), Bangalore, India

S. Choudhury, J.R. Komaragiri, P.C. Tiwari

National Institute of Science Education and Research, HBNI, Bhubaneswar, India

S. Bahinipati23 ${ }^{23}$ C. Kar, P. Mal, K. Mandal, A. Nayak ${ }^{24}$, D.K. Sahoo ${ }^{23}$, S.K. Swain 
Panjab University, Chandigarh, India

S. Bansal, S.B. Beri, V. Bhatnagar, S. Chauhan, R. Chawla, N. Dhingra, R. Gupta, A. Kaur, A. Kaur, M. Kaur, S. Kaur, R. Kumar, P. Kumari, M. Lohan, A. Mehta, K. Sandeep, S. Sharma, J.B. Singh, G. Walia

University of Delhi, Delhi, India

A. Bhardwaj, B.C. Choudhary, R.B. Garg, M. Gola, S. Keshri, Ashok Kumar, S. Malhotra, M. Naimuddin, P. Priyanka, K. Ranjan, Aashaq Shah, R. Sharma

Saha Institute of Nuclear Physics, HBNI, Kolkata, India

R. Bhardwaj ${ }^{25}$, M. Bharti, R. Bhattacharya, S. Bhattacharya, U. Bhawandeep ${ }^{25}$, D. Bhowmik, S. Dey, S. Dutt ${ }^{25}$, S. Dutta, S. Ghosh, K. Mondal, S. Nandan, A. Purohit, P.K. Rout, A. Roy, S. Roy Chowdhury, S. Sarkar, M. Sharan, B. Singh, S. Thakur ${ }^{25}$

Indian Institute of Technology Madras, Madras, India

P.K. Behera

Bhabha Atomic Research Centre, Mumbai, India

R. Chudasama, D. Dutta, V. Jha, V. Kumar, P.K. Netrakanti, L.M. Pant, P. Shukla

Tata Institute of Fundamental Research-A, Mumbai, India

T. Aziz, M.A. Bhat, S. Dugad, G.B. Mohanty, N. Sur, B. Sutar, RavindraKumar Verma

Tata Institute of Fundamental Research-B, Mumbai, India

S. Banerjee, S. Bhattacharya, S. Chatterjee, P. Das, M. Guchait, Sa. Jain, S. Karmakar, S. Kumar, M. Maity ${ }^{26}$, G. Majumder, K. Mazumdar, N. Sahoo, T. Sarkar²6

Indian Institute of Science Education and Research (IISER), Pune, India

S. Chauhan, S. Dube, V. Hegde, A. Kapoor, K. Kothekar, S. Pandey, A. Rane, S. Sharma

Institute for Research in Fundamental Sciences (IPM), Tehran, Iran

S. Chenarani ${ }^{27}$, E. Eskandari Tadavani, S.M. Etesami ${ }^{27}$, M. Khakzad, M. Mohammadi Najafabadi, M. Naseri, F. Rezaei Hosseinabadi, B. Safarzadeh ${ }^{28}$, M. Zeinali

University College Dublin, Dublin, Ireland

M. Felcini, M. Grunewald

INFN Sezione di Bari ${ }^{a}$, Università di Bari ${ }^{b}$, Politecnico di Bari ${ }^{c}$, Bari, Italy M. Abbrescia ${ }^{a, b}$, C. Calabria ${ }^{a, b}$, A. Colaleo ${ }^{a}$, D. Creanza ${ }^{a, c}$, L. Cristella ${ }^{a, b}$, N. De Filippis ${ }^{a, c}$, M. De Palma ${ }^{a, b}$, A. Di Florio ${ }^{a}, b$, F. Errico ${ }^{a, b}$, L. Fiore ${ }^{a}$, A. Gelmi ${ }^{a, b}$,

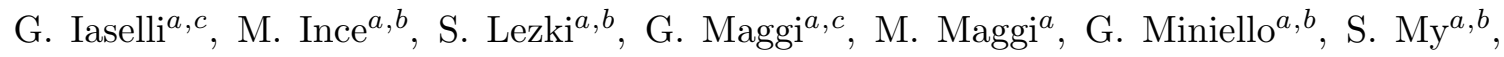
S. Nuzzo ${ }^{a, b}$, A. Pompili ${ }^{a, b}$, G. Pugliese ${ }^{a, c}$, R. Radogna ${ }^{a}$, A. Ranieri ${ }^{a}$, G. Selvaggi ${ }^{a, b}$,

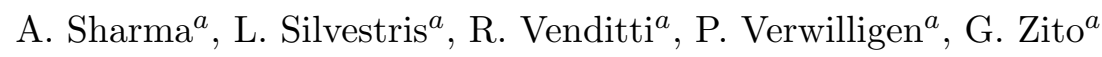

INFN Sezione di Bologna ${ }^{a}$, Università di Bologna ${ }^{b}$, Bologna, Italy

G. Abbiendi ${ }^{a}$, C. Battilana ${ }^{a, b}$, D. Bonacorsi ${ }^{a, b}$, L. Borgonovi $^{a, b}$, S. Braibant-Giacomelli ${ }^{a, b}$,

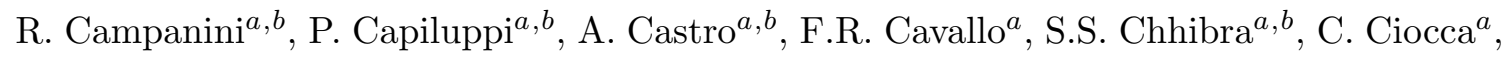

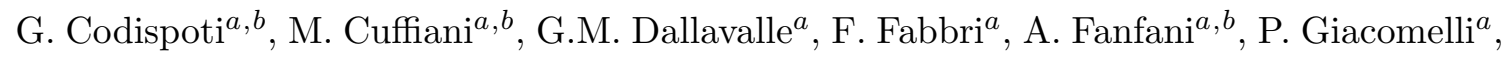
C. Grandi ${ }^{a}$, L. Guiducci ${ }^{a}, b$, F. Iemmi ${ }^{a, b}$, S. Marcellini ${ }^{a}$, G. Masetti ${ }^{a}$, A. Montanari ${ }^{a}$, 


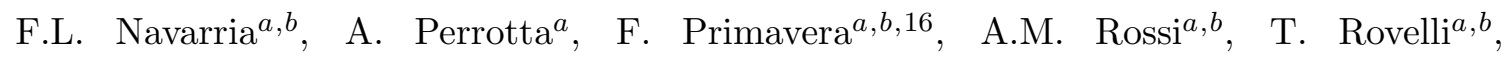
G.P. Siroli ${ }^{a, b}$, N. Tosi ${ }^{a}$

INFN Sezione di Catania ${ }^{a}$, Università di Catania ${ }^{b}$, Catania, Italy

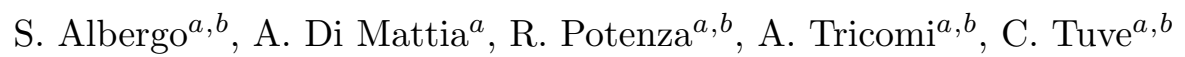

INFN Sezione di Firenze ${ }^{a}$, Università di Firenze ${ }^{b}$, Firenze, Italy

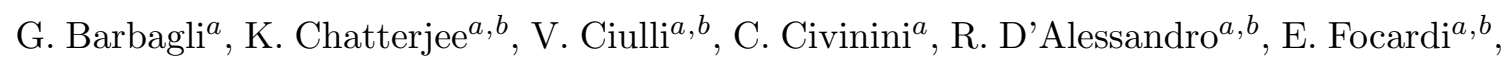

G. Latino, P. Lenzi ${ }^{a}, b$, M. Meschini ${ }^{a}$, S. Paoletti ${ }^{a}$, L. Russo $^{a, 29}$, G. Sguazzoni ${ }^{a}$, D. Strom ${ }^{a}$,

L. Viliani ${ }^{a}$

INFN Laboratori Nazionali di Frascati, Frascati, Italy

L. Benussi, S. Bianco, F. Fabbri, D. Piccolo

INFN Sezione di Genova ${ }^{a}$, Università di Genova ${ }^{b}$, Genova, Italy

F. Ferro ${ }^{a}$, F. Ravera ${ }^{a, b}$, E. Robutti $^{a}$, S. Tosi ${ }^{a, b}$

INFN Sezione di Milano-Bicocca ${ }^{a}$, Università di Milano-Bicocca ${ }^{b}$, Milano, Italy

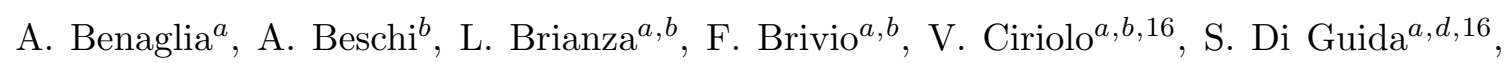

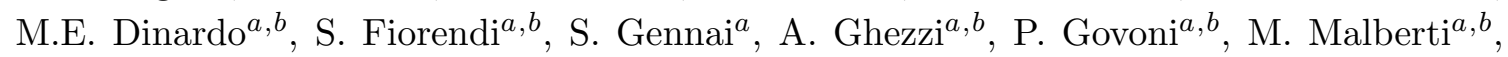
S. Malvezzi ${ }^{a}$, A. Massironi ${ }^{a, b}$, D. Menasce ${ }^{a}$, L. Moroni ${ }^{a}$, M. Paganoni $^{a}, b$, D. Pedrini ${ }^{a}$, S. Ragazzi ${ }^{a, b}$, T. Tabarelli de Fatis ${ }^{a, b}$, D. Zuolo

INFN Sezione di Napoli ${ }^{a}$, Università di Napoli 'Federico II' ${ }^{b}$, Napoli, Italy, Università della Basilicata ${ }^{c}$, Potenza, Italy, Università G. Marconi ${ }^{d}$, Roma, Italy

S. Buontempo ${ }^{a}$, N. Cavallo ${ }^{a, c}$, A. Di Crescenzo ${ }^{a, b}$, F. Fabozzi $^{a, c}$, F. Fienga $^{a}$, G. Galati $^{a}$, A.O.M. Iorio ${ }^{a, b}$, W.A. Khan ${ }^{a}$, L. $\operatorname{Lista}^{a}$, S. Meola ${ }^{a, d, 16}$, P. Paolucci ${ }^{a, 16}$, C. Sciacca ${ }^{a, b}$, E. Voevodina ${ }^{a, b}$

INFN Sezione di Padova ${ }^{a}$, Università di Padova ${ }^{b}$, Padova, Italy, Università di Trento ${ }^{c}$, Trento, Italy

P. Azzi ${ }^{a}$, N. Bacchetta ${ }^{a}$, D. Bisello ${ }^{a, b}$, A. Boletti ${ }^{a, b}$, A. Bragagnolo, R. Carlin ${ }^{a, b}$,

P. Checchia ${ }^{a}$, M. Dall'Osso ${ }^{a}, b$, P. De Castro Manzano ${ }^{a}$, T. Dorigo ${ }^{a}$, U. Dosselli ${ }^{a}$,

F. Gasparini ${ }^{a, b}$, U. Gasparini ${ }^{a, b}$, A. Gozzelino ${ }^{a}$, S. Lacaprara ${ }^{a}$, P. Lujan, M. Margoni ${ }^{a}, b$, A.T. Meneguzzo ${ }^{a, b}$, J. Pazzini ${ }^{a, b}$, P. Ronchese ${ }^{a, b}$, R. Rossin ${ }^{a, b}$, F. Simonetto ${ }^{a, b}$, A. Tiko, E. Torassa ${ }^{a}$, M. Zanetti ${ }^{a, b}$, P. Zotto ${ }^{a, b}$, G. Zumerle ${ }^{a, b}$

INFN Sezione di Pavia ${ }^{a}$, Università di Pavia ${ }^{b}$, Pavia, Italy

A. Braghieri ${ }^{a}$, A. Magnani ${ }^{a}$, P. Montagna ${ }^{a, b}$, S.P. Ratti ${ }^{a}, b$, V. $\operatorname{Re}^{a}$, M. Ressegotti ${ }^{a, b}$, C. Riccardi ${ }^{a}, b$, P. Salvini ${ }^{a}$, I. Vai $^{a, b}$, P. Vitulo ${ }^{a, b}$

INFN Sezione di Perugia ${ }^{a}$, Università di Perugia ${ }^{b}$, Perugia, Italy

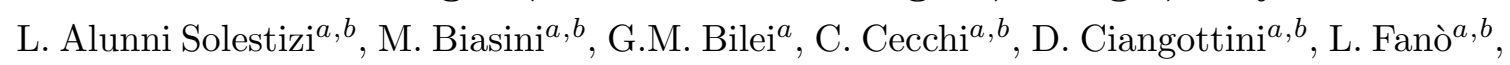
P. Lariccia ${ }^{a, b}$, R. Leonardi ${ }^{a, b}$, E. Manoni $^{a}$, G. Mantovani ${ }^{a, b}$, V. Mariani $^{a, b}$, M. Menichelli $^{a}$, A. Rossi ${ }^{a, b}$, A. Santocchia ${ }^{a, b}$, D. Spiga ${ }^{a}$ 
INFN Sezione di Pisa ${ }^{a}$, Università di Pisa ${ }^{b}$, Scuola Normale Superiore di Pisa ${ }^{c}$, Pisa, Italy

K. Androsov ${ }^{a}$, P. Azzurri ${ }^{a}$, G. Bagliesi ${ }^{a}$, L. Bianchini ${ }^{a}$, T. Boccali ${ }^{a}$, L. Borrello, R. Castaldi ${ }^{a}$, M.A. Ciocci ${ }^{a}, b$, R. Dell'Orso ${ }^{a}$, G. Fedi ${ }^{a}$, F. Fiori ${ }^{a, c}$, L. Giannini $^{a, c}$, A. Giassi $^{a}$, M.T. Grippo ${ }^{a}$, F. Ligabue ${ }^{a, c}$, E. Manca ${ }^{a, c}$, G. Mandorli ${ }^{a, c}$, A. Messineo ${ }^{a, b}$, F. Palla ${ }^{a}$,

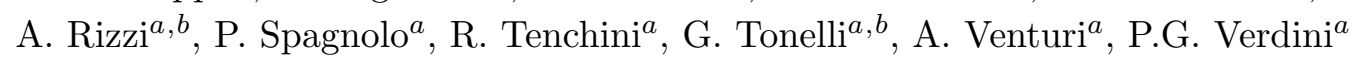

INFN Sezione di Roma ${ }^{a}$, Sapienza Università di Roma ${ }^{b}$, Rome, Italy

L. Barone ${ }^{a, b}$, F. Cavallari ${ }^{a}$, M. Cipriani ${ }^{a, b}$, N. Daci ${ }^{a}$, D. Del Re ${ }^{a, b}$, E. Di Marco ${ }^{a, b}$, M. Diemoz ${ }^{a}$, S. Gelli ${ }^{a, b}$, E. Longo ${ }^{a, b}$, B. Marzocchi ${ }^{a, b}$, P. Meridiani ${ }^{a}$, G. Organtini ${ }^{a, b}$,

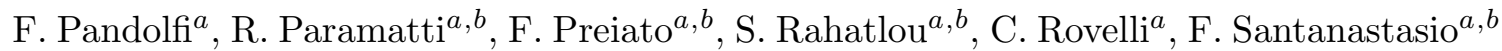

INFN Sezione di Torino ${ }^{a}$, Università di Torino ${ }^{b}$, Torino, Italy, Università del Piemonte Orientale ${ }^{c}$, Novara, Italy

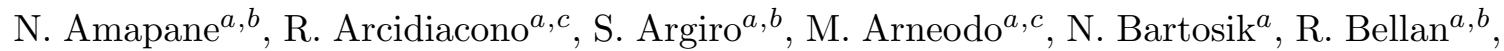

C. Biino ${ }^{a}$, N. Cartiglia $a$, F. Cenna ${ }^{a, b}$, S. Cometti, M. Costa ${ }^{a, b}$, R. Covarelli ${ }^{a, b}$,

N. Demaria ${ }^{a}$, B. Kiani ${ }^{a, b}$, C. Mariotti ${ }^{a}$, S. Maselli ${ }^{a}$, E. Migliore ${ }^{a, b}$, V. Monaco ${ }^{a, b}$,

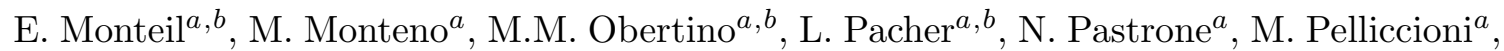
G.L. Pinna Angioni ${ }^{a, b}$, A. Romero ${ }^{a, b}$, M. Ruspa ${ }^{a, c}$, R. Sacchi ${ }^{a, b}$, K. Shchelina ${ }^{a, b}$, V. Sola ${ }^{a}$, A. Solano ${ }^{a, b}$, D. Soldi, A. Staiano ${ }^{a}$

INFN Sezione di Trieste ${ }^{a}$, Università di Trieste ${ }^{b}$, Trieste, Italy

S. Belforte ${ }^{a}$, V. Candelise ${ }^{a, b}$, M. Casarsa ${ }^{a}$, F. Cossutti ${ }^{a}$, G. Della Ricca ${ }^{a, b}$, F. Vazzoler $^{a}, b$, A. Zanetti ${ }^{a}$

Kyungpook National University

D.H. Kim, G.N. Kim, M.S. Kim, J. Lee, S. Lee, S.W. Lee, C.S. Moon, Y.D. Oh, S. Sekmen, D.C. Son, Y.C. Yang

Chonnam National University, Institute for Universe and Elementary Particles, Kwangju, Korea

H. Kim, D.H. Moon, G. Oh

Hanyang University, Seoul, Korea

J. Goh ${ }^{30}$, T.J. Kim

Korea University, Seoul, Korea

S. Cho, S. Choi, Y. Go, D. Gyun, S. Ha, B. Hong, Y. Jo, K. Lee, K.S. Lee, S. Lee, J. Lim, S.K. Park, Y. Roh

Sejong University, Seoul, Korea

H.S. Kim

Seoul National University, Seoul, Korea

J. Almond, J. Kim, J.S. Kim, H. Lee, K. Lee, K. Nam, S.B. Oh, B.C. Radburn-Smith, S.h. Seo, U.K. Yang, H.D. Yoo, G.B. Yu

University of Seoul, Seoul, Korea

D. Jeon, H. Kim, J.H. Kim, J.S.H. Lee, I.C. Park 
Sungkyunkwan University, Suwon, Korea

Y. Choi, C. Hwang, J. Lee, I. Yu

Vilnius University, Vilnius, Lithuania

V. Dudenas, A. Juodagalvis, J. Vaitkus

National Centre for Particle Physics, Universiti Malaya, Kuala Lumpur, Malaysia

I. Ahmed, Z.A. Ibrahim, M.A.B. Md Ali ${ }^{31}$, F. Mohamad Idris ${ }^{32}$, W.A.T. Wan Abdullah, M.N. Yusli, Z. Zolkapli

Universidad de Sonora (UNISON), Hermosillo, Mexico

A. Castaneda Hernandez, J.A. Murillo Quijada

Centro de Investigacion y de Estudios Avanzados del IPN, Mexico City, Mexico

H. Castilla-Valdez, E. De La Cruz-Burelo, M.C. Duran-Osuna, I. Heredia-De La Cruz ${ }^{33}$,

R. Lopez-Fernandez, J. Mejia Guisao, R.I. Rabadan-Trejo, M. Ramirez-Garcia,

G. Ramirez-Sanchez, R Reyes-Almanza, A. Sanchez-Hernandez

Universidad Iberoamericana, Mexico City, Mexico

S. Carrillo Moreno, C. Oropeza Barrera, F. Vazquez Valencia

Benemerita Universidad Autonoma de Puebla, Puebla, Mexico

J. Eysermans, I. Pedraza, H.A. Salazar Ibarguen, C. Uribe Estrada

Universidad Autónoma de San Luis Potosí, San Luis Potosí, Mexico

A. Morelos Pineda

University of Auckland, Auckland, New Zealand

D. Krofcheck

University of Canterbury, Christchurch, New Zealand

S. Bheesette, P.H. Butler

National Centre for Physics, Quaid-I-Azam University, Islamabad, Pakistan

A. Ahmad, M. Ahmad, M.I. Asghar, Q. Hassan, H.R. Hoorani, A. Saddique, M.A. Shah, M. Shoaib, M. Waqas

National Centre for Nuclear Research, Swierk, Poland

H. Bialkowska, M. Bluj, B. Boimska, T. Frueboes, M. Górski, M. Kazana, K. Nawrocki, M. Szleper, P. Traczyk, P. Zalewski

Institute of Experimental Physics, Faculty of Physics, University of Warsaw, Warsaw, Poland

K. Bunkowski, A. Byszuk ${ }^{34}$, K. Doroba, A. Kalinowski, M. Konecki, J. Krolikowski, M. Misiura, M. Olszewski, A. Pyskir, M. Walczak 
Laboratório de Instrumentação e Física Experimental de Partículas, Lisboa, Portugal

P. Bargassa, C. Beirão Da Cruz E Silva, A. Di Francesco, P. Faccioli, B. Galinhas, M. Gallinaro, J. Hollar, N. Leonardo, L. Lloret Iglesias, M.V. Nemallapudi, J. Seixas, G. Strong, O. Toldaiev, D. Vadruccio, J. Varela

\section{Joint Institute for Nuclear Research, Dubna, Russia}

S. Afanasiev, V. Alexakhin, P. Bunin, M. Gavrilenko, A. Golunov, I. Golutvin, N. Gorbounov, V. Karjavin, A. Lanev, A. Malakhov, V. Matveev ${ }^{35,36}$, P. Moisenz, V. Palichik, V. Perelygin, M. Savina, S. Shmatov, V. Smirnov, N. Voytishin, A. Zarubin

Petersburg Nuclear Physics Institute, Gatchina (St. Petersburg), Russia

V. Golovtsov, Y. Ivanov, V. Kim ${ }^{37}$, E. Kuznetsova ${ }^{38}$, P. Levchenko, V. Murzin, V. Oreshkin, I. Smirnov, D. Sosnov, V. Sulimov, L. Uvarov, S. Vavilov, A. Vorobyev

Institute for Nuclear Research, Moscow, Russia

Yu. Andreev, A. Dermenev, S. Gninenko, N. Golubev, A. Karneyeu, M. Kirsanov, N. Krasnikov, A. Pashenkov, D. Tlisov, A. Toropin

Institute for Theoretical and Experimental Physics, Moscow, Russia

V. Epshteyn, V. Gavrilov, N. Lychkovskaya, V. Popov, I. Pozdnyakov, G. Safronov, A. Spiridonov, A. Stepennov, V. Stolin, M. Toms, E. Vlasov, A. Zhokin

Moscow Institute of Physics and Technology, Moscow, Russia

T. Aushev

National Research Nuclear University 'Moscow Engineering Physics Institute' (MEPhI), Moscow, Russia

M. Chadeeva ${ }^{39}$, P. Parygin, D. Philippov, S. Polikarpov ${ }^{39}$, E. Popova, V. Rusinov

P.N. Lebedev Physical Institute, Moscow, Russia

V. Andreev, M. Azarkin ${ }^{36}$, I. Dremin ${ }^{36}$, M. Kirakosyan ${ }^{36}$, S.V. Rusakov, A. Terkulov

Skobeltsyn Institute of Nuclear Physics, Lomonosov Moscow State University, Moscow, Russia

A. Baskakov, A. Belyaev, E. Boos, M. Dubinin ${ }^{40}$, L. Dudko, A. Ershov, A. Gribushin,

V. Klyukhin, O. Kodolova, I. Lokhtin, I. Miagkov, S. Obraztsov, S. Petrushanko, V. Savrin,

A. Snigirev

Novosibirsk State University (NSU), Novosibirsk, Russia

V. Blinov ${ }^{41}$, T. Dimova ${ }^{41}$, L. Kardapoltsev ${ }^{41}$, D. Shtol ${ }^{41}$, Y. Skovpen ${ }^{41}$

State Research Center of Russian Federation, Institute for High Energy Physics of NRC "Kurchatov Institute", Protvino, Russia

I. Azhgirey, I. Bayshev, S. Bitioukov, D. Elumakhov, A. Godizov, V. Kachanov, A. Kalinin,

D. Konstantinov, P. Mandrik, V. Petrov, R. Ryutin, S. Slabospitskii, A. Sobol, S. Troshin,

N. Tyurin, A. Uzunian, A. Volkov 
National Research Tomsk Polytechnic University, Tomsk, Russia

A. Babaev, S. Baidali, V. Okhotnikov

University of Belgrade, Faculty of Physics and Vinca Institute of Nuclear Sciences, Belgrade, Serbia

P. Adzic ${ }^{42}$, P. Cirkovic, D. Devetak, M. Dordevic, J. Milosevic

Centro de Investigaciones Energéticas Medioambientales y Tecnológicas (CIEMAT), Madrid, Spain

J. Alcaraz Maestre, A. Álvarez Fernández, I. Bachiller, M. Barrio Luna, J.A. Brochero Cifuentes, M. Cerrada, N. Colino, B. De La Cruz, A. Delgado Peris, C. Fernandez Bedoya, J.P. Fernández Ramos, J. Flix, M.C. Fouz, O. Gonzalez Lopez, S. Goy Lopez, J.M. Hernandez, M.I. Josa, D. Moran, A. Pérez-Calero Yzquierdo, J. Puerta Pelayo, I. Redondo, L. Romero, M.S. Soares, A. Triossi

Universidad Autónoma de Madrid, Madrid, Spain

C. Albajar, J.F. de Trocóniz

Universidad de Oviedo, Oviedo, Spain

J. Cuevas, C. Erice, J. Fernandez Menendez, S. Folgueras, I. Gonzalez Caballero, J.R. González Fernández, E. Palencia Cortezon, V. Rodríguez Bouza, S. Sanchez Cruz, P. Vischia, J.M. Vizan Garcia

Instituto de Física de Cantabria (IFCA), CSIC-Universidad de Cantabria, Santander, Spain

I.J. Cabrillo, A. Calderon, B. Chazin Quero, J. Duarte Campderros, M. Fernandez, P.J. Fernández Manteca, A. García Alonso, J. Garcia-Ferrero, G. Gomez, A. Lopez Virto, J. Marco, C. Martinez Rivero, P. Martinez Ruiz del Arbol, F. Matorras, J. Piedra Gomez, C. Prieels, T. Rodrigo, A. Ruiz-Jimeno, L. Scodellaro, N. Trevisani, I. Vila, R. Vilar Cortabitarte

\section{CERN, European Organization for Nuclear Research, Geneva, Switzerland}

D. Abbaneo, B. Akgun, E. Auffray, P. Baillon, A.H. Ball, D. Barney, J. Bendavid, M. Bianco, A. Bocci, C. Botta, E. Brondolin, T. Camporesi, M. Cepeda, G. Cerminara, E. Chapon, Y. Chen, G. Cucciati, D. d'Enterria, A. Dabrowski, V. Daponte, A. David, A. De Roeck, N. Deelen, M. Dobson, M. Dünser, N. Dupont, A. ElliottPeisert, P. Everaerts, F. Fallavollita ${ }^{43}$, D. Fasanella, G. Franzoni, J. Fulcher, W. Funk, D. Gigi, A. Gilbert, K. Gill, F. Glege, M. Guilbaud, D. Gulhan, J. Hegeman, V. Innocente, A. Jafari, P. Janot, O. Karacheban ${ }^{19}$, J. Kieseler, A. Kornmayer, M. Krammer ${ }^{1}$, C. Lange, P. Lecoq, C. Lourenço, L. Malgeri, M. Mannelli, F. Meijers, J.A. Merlin, S. Mersi, E. Meschi, P. Milenovic ${ }^{44}$, F. Moortgat, M. Mulders, J. Ngadiuba, S. Orfanelli, L. Orsini, F. Pantaleo ${ }^{16}$, L. Pape, E. Perez, M. Peruzzi, A. Petrilli, G. Petrucciani, A. Pfeiffer, M. Pierini, F.M. Pitters, D. Rabady, A. Racz, T. Reis, G. Rolandi ${ }^{45}$, M. Rovere, H. Sakulin, C. Schäfer, C. Schwick, M. Seidel, M. Selvaggi, A. Sharma, P. Silva, P. Sphicas ${ }^{46}$, A. Stakia, J. Steggemann, M. Tosi, D. Treille, A. Tsirou, V. Veckalns ${ }^{47}$, W.D. Zeuner 
Paul Scherrer Institut, Villigen, Switzerland

L. Caminada ${ }^{48}$, K. Deiters, W. Erdmann, R. Horisberger, Q. Ingram, H.C. Kaestli, D. Kotlinski, U. Langenegger, T. Rohe, S.A. Wiederkehr

ETH Zurich - Institute for Particle Physics and Astrophysics (IPA), Zurich, Switzerland

M. Backhaus, L. Bäni, P. Berger, N. Chernyavskaya, G. Dissertori, M. Dittmar, M. Donegà, C. Dorfer, C. Grab, C. Heidegger, D. Hits, J. Hoss, T. Klijnsma, W. Lustermann, R.A. Manzoni, M. Marionneau, M.T. Meinhard, F. Micheli, P. Musella, F. Nessi-Tedaldi, J. Pata, F. Pauss, G. Perrin, L. Perrozzi, S. Pigazzini, M. Quittnat, D. Ruini, D.A. Sanz Becerra, M. Schönenberger, L. Shchutska, V.R. Tavolaro, K. Theofilatos, M.L. Vesterbacka Olsson, R. Wallny, D.H. Zhu

Universität Zürich, Zurich, Switzerland

T.K. Aarrestad, C. Amsler ${ }^{49}$, D. Brzhechko, M.F. Canelli, A. De Cosa, R. Del Burgo, S. Donato, C. Galloni, T. Hreus, B. Kilminster, I. Neutelings, D. Pinna, G. Rauco, P. Robmann, D. Salerno, K. Schweiger, C. Seitz, Y. Takahashi, A. Zucchetta

National Central University, Chung-Li, Taiwan

Y.H. Chang, K.y. Cheng, T.H. Doan, Sh. Jain, R. Khurana, C.M. Kuo, W. Lin, A. Pozdnyakov, S.S. Yu

National Taiwan University (NTU), Taipei, Taiwan

P. Chang, Y. Chao, K.F. Chen, P.H. Chen, W.-S. Hou, Arun Kumar, Y.y. Li, Y.F. Liu, R.-S. Lu, E. Paganis, A. Psallidas, A. Steen, J.f. Tsai

Chulalongkorn University, Faculty of Science, Department of Physics, Bangkok, Thailand

B. Asavapibhop, N. Srimanobhas, N. Suwonjandee

Çukurova University, Physics Department, Science and Art Faculty, Adana, Turkey

A. Bat, F. Boran, S. Cerci ${ }^{50}$, S. Damarseckin, Z.S. Demiroglu, F. Dolek, C. Dozen, I. Dumanoglu, S. Girgis, G. Gokbulut, Y. Guler, E. Gurpinar, I. $\operatorname{Hos}^{51}$, C. Isik, E.E. Kangal ${ }^{52}$, O. Kara, A. Kayis Topaksu, U. Kiminsu, M. Oglakci, G. Onengut, K. Ozdemir ${ }^{53}$, S. Ozturk ${ }^{54}$, B. Tali ${ }^{50}$, U.G. Tok, H. Topakli ${ }^{54}$, S. Turkcapar, I.S. Zorbakir, C. Zorbilmez

Middle East Technical University, Physics Department, Ankara, Turkey

B. Isildak ${ }^{55}$, G. Karapinar ${ }^{56}$, M. Yalvac, M. Zeyrek

Bogazici University, Istanbul, Turkey

I.O. Atakisi, E. Gülmez, M. Kaya ${ }^{57}$, O. Kaya ${ }^{58}$, S. Ozkorucuklu ${ }^{59}$, S. Tekten, E.A. Yetkin ${ }^{60}$

Istanbul Technical University, Istanbul, Turkey

M.N. Agaras, S. Atay, A. Cakir, K. Cankocak, Y. Komurcu, S. Sen ${ }^{61}$

Institute for Scintillation Materials of National Academy of Science of Ukraine, Kharkov, Ukraine

B. Grynyov 
National Scientific Center, Kharkov Institute of Physics and Technology, Kharkov, Ukraine

L. Levchuk

University of Bristol, Bristol, United Kingdom

F. Ball, L. Beck, J.J. Brooke, D. Burns, E. Clement, D. Cussans, O. Davignon, H. Flacher,

J. Goldstein, G.P. Heath, H.F. Heath, L. Kreczko, D.M. Newbold ${ }^{62}$, S. Paramesvaran,

B. Penning, T. Sakuma, D. Smith, V.J. Smith, J. Taylor, A. Titterton

Rutherford Appleton Laboratory, Didcot, United Kingdom

K.W. Bell, A. Belyaev ${ }^{63}$, C. Brew, R.M. Brown, D. Cieri, D.J.A. Cockerill, J.A. Coughlan,

K. Harder, S. Harper, J. Linacre, E. Olaiya, D. Petyt, C.H. Shepherd-Themistocleous,

A. Thea, I.R. Tomalin, T. Williams, W.J. Womersley

Imperial College, London, United Kingdom

G. Auzinger, R. Bainbridge, P. Bloch, J. Borg, S. Breeze, O. Buchmuller, A. Bundock, S. Casasso, D. Colling, L. Corpe, P. Dauncey, G. Davies, M. Della Negra, R. Di Maria, Y. Haddad, G. Hall, G. Iles, T. James, M. Komm, C. Laner, L. Lyons, A.-M. Magnan, S. Malik, A. Martelli, J. Nash ${ }^{64}$, A. Nikitenko ${ }^{7}$, V. Palladino, M. Pesaresi, A. Richards, A. Rose, E. Scott, C. Seez, A. Shtipliyski, G. Singh, M. Stoye, T. Strebler, S. Summers, A. Tapper, K. Uchida, T. Virdee ${ }^{16}$, N. Wardle, D. Winterbottom, J. Wright, S.C. Zenz

Brunel University, Uxbridge, United Kingdom

J.E. Cole, P.R. Hobson, A. Khan, P. Kyberd, C.K. Mackay, A. Morton, I.D. Reid, L. Teodorescu, S. Zahid

Baylor University, Waco, U.S.A.

K. Call, J. Dittmann, K. Hatakeyama, H. Liu, C. Madrid, B. Mcmaster, N. Pastika, C. Smith

Catholic University of America, Washington DC, U.S.A.

R. Bartek, A. Dominguez

The University of Alabama, Tuscaloosa, U.S.A.

A. Buccilli, S.I. Cooper, C. Henderson, P. Rumerio, C. West

Boston University, Boston, U.S.A.

D. Arcaro, T. Bose, D. Gastler, D. Rankin, C. Richardson, J. Rohlf, L. Sulak, D. Zou

Brown University, Providence, U.S.A.

G. Benelli, X. Coubez, D. Cutts, M. Hadley, J. Hakala, U. Heintz, J.M. Hogan ${ }^{65}$, K.H.M. Kwok, E. Laird, G. Landsberg, J. Lee, Z. Mao, M. Narain, S. Piperov, S. Sagir ${ }^{66}$, R. Syarif, E. Usai, D. Yu

University of California, Davis, Davis, U.S.A.

R. Band, C. Brainerd, R. Breedon, D. Burns, M. Calderon De La Barca Sanchez, M. Chertok, J. Conway, R. Conway, P.T. Cox, R. Erbacher, C. Flores, G. Funk, W. Ko, O. Kukral, R. Lander, C. Mclean, M. Mulhearn, D. Pellett, J. Pilot, S. Shalhout, M. Shi, D. Stolp, D. Taylor, K. Tos, M. Tripathi, Z. Wang, F. Zhang 
University of California, Los Angeles, U.S.A.

M. Bachtis, C. Bravo, R. Cousins, A. Dasgupta, A. Florent, J. Hauser, M. Ignatenko, N. Mccoll, S. Regnard, D. Saltzberg, C. Schnaible, V. Valuev

University of California, Riverside, Riverside, U.S.A.

E. Bouvier, K. Burt, R. Clare, J.W. Gary, S.M.A. Ghiasi Shirazi, G. Hanson, G. Karapostoli, E. Kennedy, F. Lacroix, O.R. Long, M. Olmedo Negrete, M.I. Paneva, W. Si, L. Wang, H. Wei, S. Wimpenny, B.R. Yates

University of California, San Diego, La Jolla, U.S.A.

J.G. Branson, S. Cittolin, M. Derdzinski, R. Gerosa, D. Gilbert, B. Hashemi, A. Holzner, D. Klein, G. Kole, V. Krutelyov, J. Letts, M. Masciovecchio, D. Olivito, S. Padhi, M. Pieri, M. Sani, V. Sharma, S. Simon, M. Tadel, A. Vartak, S. Wasserbaech ${ }^{67}$, J. Wood, F. Würthwein, A. Yagil, G. Zevi Della Porta

University of California, Santa Barbara - Department of Physics, Santa Barbara, U.S.A.

N. Amin, R. Bhandari, J. Bradmiller-Feld, C. Campagnari, M. Citron, A. Dishaw,

V. Dutta, M. Franco Sevilla, L. Gouskos, R. Heller, J. Incandela, A. Ovcharova, H. Qu,

J. Richman, D. Stuart, I. Suarez, S. Wang, J. Yoo

California Institute of Technology, Pasadena, U.S.A.

D. Anderson, A. Bornheim, J.M. Lawhorn, H.B. Newman, T.Q. Nguyen, M. Spiropulu, J.R. Vlimant, R. Wilkinson, S. Xie, Z. Zhang, R.Y. Zhu

Carnegie Mellon University, Pittsburgh, U.S.A.

M.B. Andrews, T. Ferguson, T. Mudholkar, M. Paulini, M. Sun, I. Vorobiev, M. Weinberg

University of Colorado Boulder, Boulder, U.S.A.

J.P. Cumalat, W.T. Ford, F. Jensen, A. Johnson, M. Krohn, S. Leontsinis, E. MacDonald, T. Mulholland, K. Stenson, K.A. Ulmer, S.R. Wagner

Cornell University, Ithaca, U.S.A.

J. Alexander, J. Chaves, Y. Cheng, J. Chu, A. Datta, K. Mcdermott, N. Mirman, J.R. Patterson, D. Quach, A. Rinkevicius, A. Ryd, L. Skinnari, L. Soffi, S.M. Tan, Z. Tao, J. Thom, J. Tucker, P. Wittich, M. Zientek

Fermi National Accelerator Laboratory, Batavia, U.S.A.

S. Abdullin, M. Albrow, M. Alyari, G. Apollinari, A. Apresyan, A. Apyan, S. Banerjee, L.A.T. Bauerdick, A. Beretvas, J. Berryhill, P.C. Bhat, G. Bolla ${ }^{\dagger}$, K. Burkett, J.N. Butler, A. Canepa, G.B. Cerati, H.W.K. Cheung, F. Chlebana, M. Cremonesi, J. Duarte, V.D. Elvira, J. Freeman, Z. Gecse, E. Gottschalk, L. Gray, D. Green, S. Grünendahl, O. Gutsche, J. Hanlon, R.M. Harris, S. Hasegawa, J. Hirschauer, Z. Hu, B. Jayatilaka, S. Jindariani, M. Johnson, U. Joshi, B. Klima, M.J. Kortelainen, B. Kreis, S. Lammel, D. Lincoln, R. Lipton, M. Liu, T. Liu, J. Lykken, K. Maeshima, J.M. Marraffino, D. Mason, P. McBride, P. Merkel, S. Mrenna, S. Nahn, V. O’Dell, K. Pedro, C. Pena, O. Prokofyev, G. Rakness, L. Ristori, A. Savoy-Navarro ${ }^{68}$, B. Schneider, E. Sexton-Kennedy, A. Soha, 
W.J. Spalding, L. Spiegel, S. Stoynev, J. Strait, N. Strobbe, L. Taylor, S. Tkaczyk, N.V. Tran, L. Uplegger, E.W. Vaandering, C. Vernieri, M. Verzocchi, R. Vidal, M. Wang, H.A. Weber, A. Whitbeck

University of Florida, Gainesville, U.S.A.

D. Acosta, P. Avery, P. Bortignon, D. Bourilkov, A. Brinkerhoff, L. Cadamuro, A. Carnes, M. Carver, D. Curry, R.D. Field, S.V. Gleyzer, B.M. Joshi, J. Konigsberg, A. Korytov, P. Ma, K. Matchev, H. Mei, G. Mitselmakher, K. Shi, D. Sperka, J. Wang, S. Wang

Florida International University, Miami, U.S.A.

Y.R. Joshi, S. Linn

Florida State University, Tallahassee, U.S.A.

A. Ackert, T. Adams, A. Askew, S. Hagopian, V. Hagopian, K.F. Johnson, T. Kolberg, G. Martinez, T. Perry, H. Prosper, A. Saha, V. Sharma, R. Yohay

Florida Institute of Technology, Melbourne, U.S.A.

M.M. Baarmand, V. Bhopatkar, S. Colafranceschi, M. Hohlmann, D. Noonan, M. Rahmani, T. Roy, F. Yumiceva

University of Illinois at Chicago (UIC), Chicago, U.S.A.

M.R. Adams, L. Apanasevich, D. Berry, R.R. Betts, R. Cavanaugh, X. Chen, S. Dittmer, O. Evdokimov, C.E. Gerber, D.A. Hangal, D.J. Hofman, K. Jung, J. Kamin, C. Mills, I.D. Sandoval Gonzalez, M.B. Tonjes, N. Varelas, H. Wang, X. Wang, Z. Wu, J. Zhang

The University of Iowa, Iowa City, U.S.A.

M. Alhusseini, B. Bilki ${ }^{69}$, W. Clarida, K. Dilsiz ${ }^{70}$, S. Durgut, R.P. Gandrajula, M. Haytmyradov, V. Khristenko, J.-P. Merlo, A. Mestvirishvili, A. Moeller, J. Nachtman, H. Ogul ${ }^{71}$, Y. Onel, F. Ozok ${ }^{72}$, A. Penzo, C. Snyder, E. Tiras, J. Wetzel

Johns Hopkins University, Baltimore, U.S.A.

B. Blumenfeld, A. Cocoros, N. Eminizer, D. Fehling, L. Feng, A.V. Gritsan, W.T. Hung, P. Maksimovic, J. Roskes, U. Sarica, M. Swartz, M. Xiao, C. You

The University of Kansas, Lawrence, U.S.A.

A. Al-bataineh, P. Baringer, A. Bean, S. Boren, J. Bowen, A. Bylinkin, J. Castle, S. Khalil,

A. Kropivnitskaya, D. Majumder, W. Mcbrayer, M. Murray, C. Rogan, S. Sanders, E. Schmitz, J.D. Tapia Takaki, Q. Wang

\section{Kansas State University, Manhattan, U.S.A.}

S. Duric, A. Ivanov, K. Kaadze, D. Kim, Y. Maravin, D.R. Mendis, T. Mitchell, A. Modak, A. Mohammadi, L.K. Saini, N. Skhirtladze

Lawrence Livermore National Laboratory, Livermore, U.S.A.

F. Rebassoo, D. Wright 
University of Maryland, College Park, U.S.A.

A. Baden, O. Baron, A. Belloni, S.C. Eno, Y. Feng, C. Ferraioli, N.J. Hadley, S. Jabeen, G.Y. Jeng, R.G. Kellogg, J. Kunkle, A.C. Mignerey, F. Ricci-Tam, Y.H. Shin, A. Skuja, S.C. Tonwar, K. Wong

\section{Massachusetts Institute of Technology, Cambridge, U.S.A.}

D. Abercrombie, B. Allen, V. Azzolini, A. Baty, G. Bauer, R. Bi, S. Brandt, W. Busza, I.A. Cali, M. D'Alfonso, Z. Demiragli, G. Gomez Ceballos, M. Goncharov, P. Harris, D. Hsu, M. Hu, Y. Iiyama, G.M. Innocenti, M. Klute, D. Kovalskyi, Y.-J. Lee, P.D. Luckey, B. Maier, A.C. Marini, C. Mcginn, C. Mironov, S. Narayanan, X. Niu, C. Paus, C. Roland, G. Roland, G.S.F. Stephans, K. Sumorok, K. Tatar, D. Velicanu, J. Wang, T.W. Wang, B. Wyslouch, S. Zhaozhong

University of Minnesota, Minneapolis, U.S.A.

A.C. Benvenuti, R.M. Chatterjee, A. Evans, P. Hansen, S. Kalafut, Y. Kubota, Z. Lesko, J. Mans, S. Nourbakhsh, N. Ruckstuhl, R. Rusack, J. Turkewitz, M.A. Wadud

University of Mississippi, Oxford, U.S.A.

J.G. Acosta, S. Oliveros

University of Nebraska-Lincoln, Lincoln, U.S.A.

E. Avdeeva, K. Bloom, D.R. Claes, C. Fangmeier, F. Golf, R. Gonzalez Suarez, R. Kamalieddin, I. Kravchenko, J. Monroy, J.E. Siado, G.R. Snow, B. Stieger

State University of New York at Buffalo, Buffalo, U.S.A.

A. Godshalk, C. Harrington, I. Iashvili, A. Kharchilava, D. Nguyen, A. Parker, S. Rappoccio, B. Roozbahani

Northeastern University, Boston, U.S.A.

G. Alverson, E. Barberis, C. Freer, A. Hortiangtham, D.M. Morse, T. Orimoto, R. Teixeira De Lima, T. Wamorkar, B. Wang, A. Wisecarver, D. Wood

Northwestern University, Evanston, U.S.A.

S. Bhattacharya, O. Charaf, K.A. Hahn, N. Mucia, N. Odell, M.H. Schmitt, K. Sung, M. Trovato, M. Velasco

University of Notre Dame, Notre Dame, U.S.A.

R. Bucci, N. Dev, M. Hildreth, K. Hurtado Anampa, C. Jessop, D.J. Karmgard, N. Kellams, K. Lannon, W. Li, N. Loukas, N. Marinelli, F. Meng, C. Mueller, Y. Musienko ${ }^{35}$, M. Planer, A. Reinsvold, R. Ruchti, P. Siddireddy, G. Smith, S. Taroni, M. Wayne, A. Wightman, M. Wolf, A. Woodard

The Ohio State University, Columbus, U.S.A.

J. Alimena, L. Antonelli, B. Bylsma, L.S. Durkin, S. Flowers, B. Francis, A. Hart, C. Hill, W. Ji, T.Y. Ling, W. Luo, B.L. Winer, H.W. Wulsin 
Princeton University, Princeton, U.S.A.

S. Cooperstein, P. Elmer, J. Hardenbrook, P. Hebda, S. Higginbotham, A. Kalogeropoulos,

D. Lange, M.T. Lucchini, J. Luo, D. Marlow, K. Mei, I. Ojalvo, J. Olsen, C. Palmer, P. Piroué, J. Salfeld-Nebgen, D. Stickland, C. Tully

University of Puerto Rico, Mayaguez, U.S.A.

S. Malik, S. Norberg

Purdue University, West Lafayette, U.S.A.

A. Barker, V.E. Barnes, S. Das, L. Gutay, M. Jones, A.W. Jung, A. Khatiwada, B. Mahakud, D.H. Miller, N. Neumeister, C.C. Peng, H. Qiu, J.F. Schulte, J. Sun, F. Wang, R. Xiao, W. Xie

Purdue University Northwest, Hammond, U.S.A.

T. Cheng, J. Dolen, N. Parashar

Rice University, Houston, U.S.A.

Z. Chen, K.M. Ecklund, S. Freed, F.J.M. Geurts, M. Kilpatrick, W. Li, B. Michlin, B.P. Padley, J. Roberts, J. Rorie, W. Shi, Z. Tu, J. Zabel, A. Zhang

University of Rochester, Rochester, U.S.A.

A. Bodek, P. de Barbaro, R. Demina, Y.t. Duh, J.L. Dulemba, C. Fallon, T. Ferbel, M. Galanti, A. Garcia-Bellido, J. Han, O. Hindrichs, A. Khukhunaishvili, K.H. Lo, P. Tan, R. Taus, M. Verzetti

Rutgers, The State University of New Jersey, Piscataway, U.S.A.

A. Agapitos, J.P. Chou, Y. Gershtein, T.A. Gómez Espinosa, E. Halkiadakis, M. Heindl, E. Hughes, S. Kaplan, R. Kunnawalkam Elayavalli, S. Kyriacou, A. Lath, R. Montalvo, K. Nash, M. Osherson, H. Saka, S. Salur, S. Schnetzer, D. Sheffield, S. Somalwar, R. Stone, S. Thomas, P. Thomassen, M. Walker

University of Tennessee, Knoxville, U.S.A.

A.G. Delannoy, J. Heideman, G. Riley, S. Spanier, K. Thapa

Texas A\&M University, College Station, U.S.A.

O. Bouhali ${ }^{73}$, A. Celik, M. Dalchenko, M. De Mattia, A. Delgado, S. Dildick, R. Eusebi, J. Gilmore, T. Huang, T. Kamon ${ }^{74}$, S. Luo, R. Mueller, R. Patel, A. Perloff, L. Perniè, D. Rathjens, A. Safonov

\section{Texas Tech University, Lubbock, U.S.A.}

N. Akchurin, J. Damgov, F. De Guio, P.R. Dudero, S. Kunori, K. Lamichhane, S.W. Lee, T. Mengke, S. Muthumuni, T. Peltola, S. Undleeb, I. Volobouev, Z. Wang

Vanderbilt University, Nashville, U.S.A.

S. Greene, A. Gurrola, R. Janjam, W. Johns, C. Maguire, A. Melo, H. Ni, K. Padeken, J.D. Ruiz Alvarez, P. Sheldon, S. Tuo, J. Velkovska, M. Verweij, Q. Xu 


\section{University of Virginia, Charlottesville, U.S.A.}

M.W. Arenton, P. Barria, B. Cox, R. Hirosky, M. Joyce, A. Ledovskoy, H. Li, C. Neu, T. Sinthuprasith, Y. Wang, E. Wolfe, F. Xia

Wayne State University, Detroit, U.S.A.

R. Harr, P.E. Karchin, N. Poudyal, J. Sturdy, P. Thapa, S. Zaleski

University of Wisconsin - Madison, Madison, WI, U.S.A.

M. Brodski, J. Buchanan, C. Caillol, D. Carlsmith, S. Dasu, L. Dodd, B. Gomber, M. Grothe, M. Herndon, A. Hervé, U. Hussain, P. Klabbers, A. Lanaro, A. Levine, K. Long, R. Loveless, T. Ruggles, A. Savin, N. Smith, W.H. Smith, N. Woods

$\dagger$ : Deceased

1: Also at Vienna University of Technology, Vienna, Austria

2: Also at IRFU, CEA, Université Paris-Saclay, Gif-sur-Yvette, France

3: Also at Universidade Estadual de Campinas, Campinas, Brazil

4: Also at Federal University of Rio Grande do Sul, Porto Alegre, Brazil

5: Also at Université Libre de Bruxelles, Bruxelles, Belgium

6: Also at University of Chinese Academy of Sciences, Beijing, China

7: Also at Institute for Theoretical and Experimental Physics, Moscow, Russia

8: Also at Joint Institute for Nuclear Research, Dubna, Russia

9: Also at Suez University, Suez, Egypt

10: Now at British University in Egypt, Cairo, Egypt

11: Also at Zewail City of Science and Technology, Zewail, Egypt

12: Now at Helwan University, Cairo, Egypt

13: Also at Department of Physics, King Abdulaziz University, Jeddah, Saudi Arabia

14: Also at Université de Haute Alsace, Mulhouse, France

15: Also at Skobeltsyn Institute of Nuclear Physics, Lomonosov Moscow State University, Moscow, Russia

16: Also at CERN, European Organization for Nuclear Research, Geneva, Switzerland

17: Also at RWTH Aachen University, III. Physikalisches Institut A, Aachen, Germany

18: Also at University of Hamburg, Hamburg, Germany

19: Also at Brandenburg University of Technology, Cottbus, Germany

20: Also at MTA-ELTE Lendület CMS Particle and Nuclear Physics Group, Eötvös Loránd University, Budapest, Hungary

21: Also at Institute of Nuclear Research ATOMKI, Debrecen, Hungary

22: Also at Institute of Physics, University of Debrecen, Debrecen, Hungary

23: Also at Indian Institute of Technology Bhubaneswar, Bhubaneswar, India

24: Also at Institute of Physics, Bhubaneswar, India

25: Also at Shoolini University, Solan, India

26: Also at University of Visva-Bharati, Santiniketan, India

27: Also at Isfahan University of Technology, Isfahan, Iran

28: Also at Plasma Physics Research Center, Science and Research Branch, Islamic Azad University, Tehran, Iran

29: Also at Università degli Studi di Siena, Siena, Italy

30: Also at Kyunghee University, Seoul, Korea

31: Also at International Islamic University of Malaysia, Kuala Lumpur, Malaysia 
32: Also at Malaysian Nuclear Agency, MOSTI, Kajang, Malaysia

33: Also at Consejo Nacional de Ciencia y Tecnología, Mexico city, Mexico

34: Also at Warsaw University of Technology, Institute of Electronic Systems, Warsaw, Poland

35: Also at Institute for Nuclear Research, Moscow, Russia

36: Now at National Research Nuclear University 'Moscow Engineering Physics Institute' (MEPhI), Moscow, Russia

37: Also at St. Petersburg State Polytechnical University, St. Petersburg, Russia

38: Also at University of Florida, Gainesville, U.S.A.

39: Also at P.N. Lebedev Physical Institute, Moscow, Russia

40: Also at California Institute of Technology, Pasadena, U.S.A.

41: Also at Budker Institute of Nuclear Physics, Novosibirsk, Russia

42: Also at Faculty of Physics, University of Belgrade, Belgrade, Serbia

43: Also at INFN Sezione di Pavia ${ }^{a}$, Università di Pavia ${ }^{b}$, Pavia, Italy

44: Also at University of Belgrade, Faculty of Physics and Vinca Institute of Nuclear Sciences, Belgrade, Serbia

45: Also at Scuola Normale e Sezione dell'INFN, Pisa, Italy

46: Also at National and Kapodistrian University of Athens, Athens, Greece

47: Also at Riga Technical University, Riga, Latvia

48: Also at Universität Zürich, Zurich, Switzerland

49: Also at Stefan Meyer Institute for Subatomic Physics (SMI), Vienna, Austria

50: Also at Adiyaman University, Adiyaman, Turkey

51: Also at Istanbul Aydin University, Istanbul, Turkey

52: Also at Mersin University, Mersin, Turkey

53: Also at Piri Reis University, Istanbul, Turkey

54: Also at Gaziosmanpasa University, Tokat, Turkey

55: Also at Ozyegin University, Istanbul, Turkey

56: Also at Izmir Institute of Technology, Izmir, Turkey

57: Also at Marmara University, Istanbul, Turkey

58: Also at Kafkas University, Kars, Turkey

59: Also at Istanbul University, Faculty of Science, Istanbul, Turkey

60: Also at Istanbul Bilgi University, Istanbul, Turkey

61: Also at Hacettepe University, Ankara, Turkey

62: Also at Rutherford Appleton Laboratory, Didcot, United Kingdom

63: Also at School of Physics and Astronomy, University of Southampton, Southampton, United Kingdom

64: Also at Monash University, Faculty of Science, Clayton, Australia

65: Also at Bethel University, St. Paul, U.S.A.

66: Also at Karamanoğlu Mehmetbey University, Karaman, Turkey

67: Also at Utah Valley University, Orem, U.S.A.

68: Also at Purdue University, West Lafayette, U.S.A.

69: Also at Beykent University, Istanbul, Turkey

70: Also at Bingol University, Bingol, Turkey

71: Also at Sinop University, Sinop, Turkey

72: Also at Mimar Sinan University, Istanbul, Istanbul, Turkey

73: Also at Texas A\&M University at Qatar, Doha, Qatar

74: Also at Kyungpook National University, Daegu, Korea 